\title{
Vertical Vortex Development in Hurricane Michael (2018) during Rapid Intensification
}

\author{
Alexander J. DesRosiers, ${ }^{\mathrm{a}}$ Michael M. Bell, ${ }^{\mathrm{a}}$ AND Ting-Yu ChA ${ }^{\mathrm{a}}$

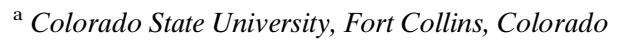

(Manuscript received 29 April 2021, in final form 5 October 2021)

\begin{abstract}
The landfall of Hurricane Michael (2018) at category-5 intensity occurred after rapid intensification (RI) spanning much of the storm's lifetime. Four Hurricane Hunter aircraft missions observed the RI period with tail Doppler radar (TDR). Data from each of the 14 aircraft passes through the storm were quality controlled via a combination of interactive and machine-learning techniques. TDR data from each pass were synthesized using the Spline Analysis at Mesoscale Utilizing Radar and Aircraft Instrumentation (SAMURAI) variational wind retrieval technique to yield threedimensional kinematic fields of the storm to examine inner-core processes during RI. Vorticity and angular momentum increased and concentrated in the eyewall region. A vorticity budget analysis indicates that the tendencies became more axisymmetric over time. In this study, we focus in particular on how the eyewall vorticity tower builds vertically into the upper levels. Horizontal vorticity associated with the vertical gradient of tangential wind was tilted into the vertical by the eyewall updraft to yield a positive vertical vorticity tendency inward atop the existing vorticity tower, which is further developed locally upward and outward along the sloped eyewall through advection and stretching. Observed maintenance of thermal wind balance from a thermodynamic retrieval shows evidence of a strengthening warm core, which aided in lowering surface pressure and further contributed to the efficient intensification in the latter stages of this RI event.
\end{abstract}

KEYWORDS: Tropical cyclones; Vorticity; Radars/radar observations

\section{Introduction}

As large strides have been made in improving track forecasts by NOAA's National Hurricane Center (NHC), intensity forecast improvements have lagged behind. Improvements have been made in intensity forecasts in recent years but were slowest to occur in the 24-48-h forecast periods (DeMaria et al. 2014). The NHC defines rapid intensification (RI) as an increase in the maximum sustained winds of a tropical cyclone (TC) of at least $30 \mathrm{kt}\left(1 \mathrm{kt} \approx 0.51 \mathrm{~m} \mathrm{~s}^{-1}\right)$ in a 24-h period (Kaplan and DeMaria 2003; Kaplan et al. 2010). More recent advancements in modeling, development of consensus forecast aids and RI guidance, and skill of NHC forecasters led to an increase in the probability of detection of short-term RI events but no notable improvement in false-alarm rates (Cangialosi et al. 2020). From 1989 to 2018, RI was successfully predicted approximately $3 \%$ of the time in the Atlantic Ocean basin (Trabing and Bell 2020). Further improvements to intensity forecasting depend in part on better prediction of rapid intensity changes. Poor prediction of rapid intensity changes causes larger errors, explaining roughly $20 \%$ in the yearly mean absolute errors in intensity forecasts over the same period (Trabing and Bell 2020). Hendricks et al. (2010) quantified the impact of environmental factors on TC intensity change and concluded that RI is mostly controlled by internal dynamical processes in similarly favorable environments.

TC intensification is a cooperative process between cloud-scale moist convection and cyclone-scale circulation that allows heat and moisture from the ocean to be utilized for TC growth and maintenance (Ooyama 1969). Increasing net vertical mass flux in

Cha's current affiliation: Department of Atmospheric Science, Colorado State University, Fort Collins, Colorado.

Corresponding author: Alexander J. DesRosiers, adesros@rams. colostate.edu the inner core due to the secondary circulation composed of inflow at the surface, upward motion in the eyewall, and outflow aloft is an essential component of strengthening (Ooyama 1982). More current investigation of TC intensification has placed emphasis on the mesoscale within the storm. In the beginning of the TC life cycle, intense individual convective elements termed vortical hot towers (VHTs) precondition the TC environment by tilting and stretching vorticity (Hendricks et al. 2004). VHTs facilitate convergence of angular momentum in the lower levels and latent heat release as a source of warming near the disturbance center. Genesis can be accomplished by the merger and axisymmetrization of VHTs, which can create a system-scale circulation (Montgomery et al. 2006). Discrete convective elements remain important to the TC intensification process as it progresses toward RI. Many deep convective towers locally amplify rotation in the TC at the onset of RI but decrease in number as RI proceeds (Van Sang et al. 2008). A composite study of aircraft reconnaissance grouped observations in intensifying and steady state hurricanes to identify differences between the groups (Rogers et al. 2013). Anomalous convective updrafts with high local vertical velocities termed convective bursts $(\mathrm{CBs})$ occurred with greater frequency and were preferentially located within the radius of maximum wind (RMW) in the intensifying storms. Convection within the RMW has been shown in theoretical work to favor RI as well (Vigh and Schubert 2009).

Simulation of RI indicated a synergistic relationship between $\mathrm{CBs}$ and the background secondary circulation. Individual CBs can enhance mass flux prior to RI before the increased mass flux is accomplished by the average vertical motion in the inner core (Rogers 2010). The greatest contribution to intensification comes from the axisymmetric projection of the heating (Nolan et al. 2007) that is collocated with the eyewall updraft in a TC inner core. The transition in intensification from discrete CBs to a more axisymmetric secondary circulation is also hinted at in 
the composite study of aircraft observations. Intensifying storms in the study exhibited ring-like vorticity focused inside the RMW and stronger axisymmetric projections of upward motion (Rogers et al. 2013). Updraft mass flux also peaked at higher altitudes and decreased less rapidly with height in the intensifying storm composite observations when compared with steady state. A preference toward mass flux peaks at greater elevation suggests the vertical extent of inner-core eyewall convection may also be important to intensification. A composite study of microwave brightness temperatures found that during RI events a peak in $37-\mathrm{GHz}$ anomalies, which are sensitive to increased liquid water path from precipitation below the freezing level, preceded peaks at $85 \mathrm{GHz}$, mainly due to scattering by ice water path above the freezing level (Fischer et al. 2018). The composited satellite observations lend further credence to the idea of a progression toward deeper inner-core convection during RI.

Previous studies have examined processes in the upper troposphere during RI, but there is a lack of consensus on their role. Simulation of RI in Hurricane Wilma (2005) suggested the importance of the formation and location of the upper-level warm core strengthened through subsidence from deep asymmetric CBs (Chen and Zhang 2013). A double warm core structure has also been simulated with the strongest warming located in the midlevel maxima (Stern and Zhang 2016). Although both studies show upper-level warming, with differing importance assigned to it, arguments have been made that the height of the warm core is not related to intensity (Stern and Nolan 2012). However, in general warming at higher altitude in the atmosphere can be more efficient at lowering surface pressure through hydrostatic arguments (Hirschberg and Fritsch 1993).

More-detailed inner-core information is required to improve skill in the prediction of RI (Kaplan et al. 2010). Research flights utilizing tail Doppler radar (TDR) have been a crucial asset to furthering our understanding of inner-core processes in TCs. Analysis of TDR data collected during the RI of Hurricane Patricia (2015) documented an intense axisymmetric inner core, which extended deep into the troposphere (Rogers et al. 2017). Analysis of the axisymmetric potential vorticity (PV) structure suggests that the vortex was able to concentrate PV efficiently along angular momentum surfaces just inward of the RMW during RI over a deep layer through diabatic heating (Martinez et al. 2019). Idealized numerical simulations also provide support for the idea that a deeper vortex promotes faster intensification of a TC (Peng and Fang 2021).

Stern and Nolan (2009) used TDR data and theory to evaluate the role of vertical structure of the tangential wind field in intensification of a TC, but questions remained about decay of the tangential wind field in the upper levels where past observations had been more sparse. A combination of a 14$\mathrm{dB}$ increase in sensitivity (Aircraft Operations Center 2016) of the TDR flying aboard the NOAA P3 Hurricane Hunter Aircraft during the 2018 Atlantic hurricane season and the excellent aircraft data coverage during Hurricane Michael allow herein for detailed observation of the upper levels during RI. The current study builds on prior work investigating the asymmetric contributions to Hurricane Michael's RI deduced from radar observations (Cha et al. 2020), and the relationship between sea surface temperature gradients from oceanic eddies and shear induced asymmetry (Wadler et al. 2020). This study is focused on investigating the axisymmetric dynamical aspects of vertical growth of the vorticity tower of Hurricane Michael and inferred connections of upper-level processes to RI of the near-surface wind field. The expansive temporal coverage of aircraft observations collected by the NOAA Aircraft Operations Center provided an excellent dataset with which to examine these facets of the RI process in Hurricane Michael.

\section{Synopsis of Hurricane Michael and observation periods}

The tropical depression that developed into Hurricane Michael was first designated by the NHC on 0600 UTC 7 October about $130 \mathrm{n} \mathrm{mi}(1 \mathrm{n} \mathrm{mi} \approx 1.85 \mathrm{~km})$ south of Cozumel, Mexico (Beven et al. 2019). The genesis occurred in a large area of disturbed weather in the western Caribbean Sea. The disturbance was composed of the remnants of Tropical Storm Kirk, a separate surface low, and a larger cyclonic gyre. Moderate vertical wind shear present in the surrounding environment failed to prevent intensification of the system and RI began immediately. Michael attained tropical storm status $6 \mathrm{~h}$ after formation and hurricane status a day later (Fig. 1a).

A brief pause in intensification took place on 8 October near the western tip of Cuba as Michael entered the Southern Gulf of Mexico via the Yucatan Channel. A 24-h intensification rate centered on this lull is below the RI threshold that was otherwise met from designation as a tropical storm onward through landfall. Reconnaissance performed by the NOAA P3 research aircraft began during this time, and the initial flight (yellow; Fig. 1a) sampled Michael at category- 2 intensity with maximum sustained winds of $85 \mathrm{kt}$. High-resolution ensemble modeling of Hurricane Michael initialized at 1800 UTC 7 October found no statistical significance in structure metrics between the stronger and weaker ensemble member groups $12 \mathrm{~h}$ into simulation with differences in the groups emerging later (Hazelton et al. 2020). These results indicate that beginning to sample the storm on 8 October was well timed to capture structural contributions to RI. Under the steering influence of a ridge and midlatitude shortwave trough, Michael took a general northward track through the Gulf of Mexico. Intensification resumed on 9 October and continued until landfall. Two aircraft missions (orange and blue; Fig. 1a) on 9 October captured the storm during intensification from category-2 to category-4 status. A final NOAA mission (green; Fig. 1a) on 10 October observed category-4 Hurricane Michael as it continued to intensify on approach to land, just before the final designation of category-5 intensity near landfall. Details of the time spent in storm by the P3, center fix times of passes through the storm, and their temporal position relative to the storm's changing intensity are shown in Fig. 1.

Landfall took place at 1730 UTC 10 October near Tyndall Air Force Base in the Florida Panhandle. Minimum central pressure was recorded at $919 \mathrm{hPa}$, with maximum sustained winds estimated at $140 \mathrm{kt}$. Michael became the fourth storm 

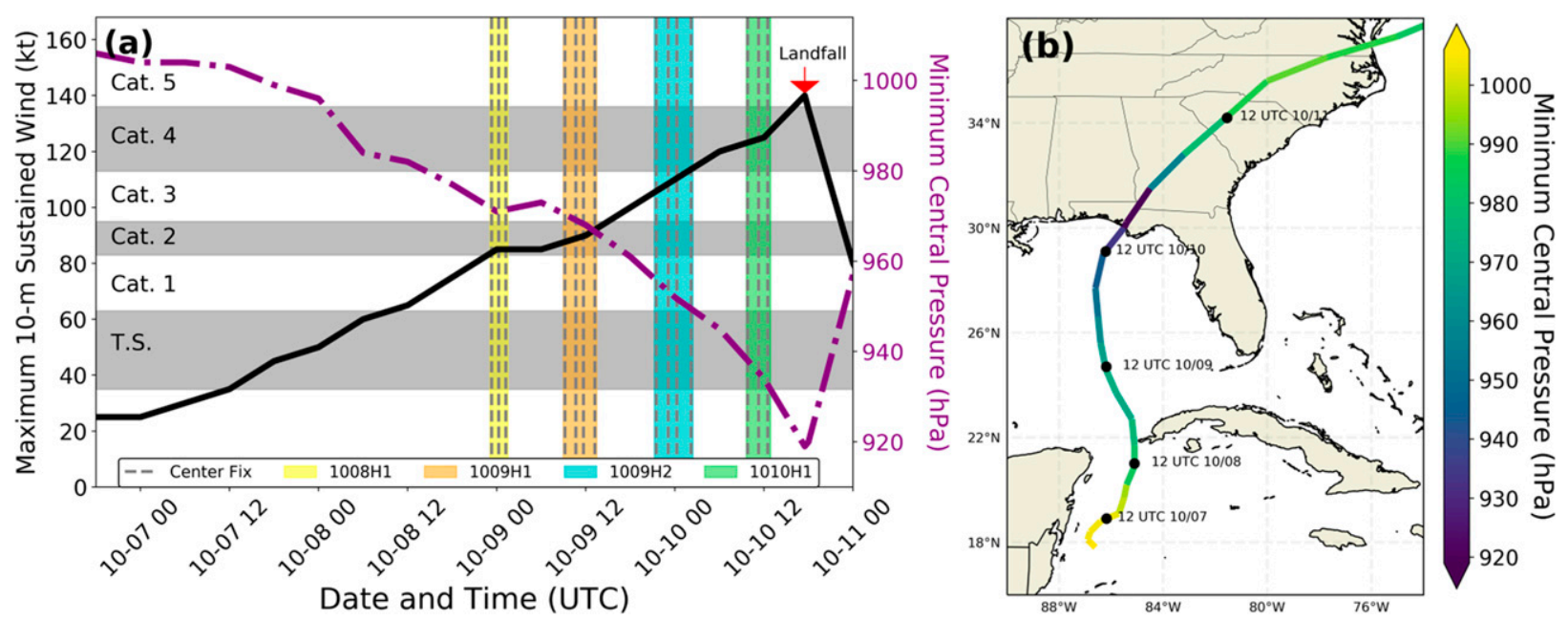

FIG. 1. (a) NHC best-track maximum sustained 10-m wind speed (kt) and minimum central pressure (hPa) from genesis through shortly after landfall. Observation periods by the P3 research aircraft are highlighted with embedded vertical dashed lines indicating the time at which the aircraft reached the center during each pass through Hurricane Michael. (b) Track of the storm from genesis through exiting the United States, with minimum central pressure shaded.

on record to make landfall in the mainland United States at category-5 intensity. The storm caused extensive damage, most notably in the immediate coastal communities of Panama City and Mexico Beach, the latter of which was impacted by catastrophic storm surge reaching up to $14 \mathrm{ft}(1 \mathrm{ft}$ $\approx 30.5 \mathrm{~cm}$ ) above ground level. The storm's damaging trek continued inland across the southeastern United States as Michael maintained category-3 intensity while crossing the southwest Georgia border, making it the first major hurricane to impact the state since 1890 (National Weather Service 2019). The storm tracked through South Carolina, North Carolina, and Virginia while undergoing extratropical transition before re-emerging over the Atlantic Ocean (Fig. 1b). Hurricane Michael caused $\$ 25$ billion (U.S. dollars) in damage in the United States and was directly responsible for 16 deaths (Beven et al. 2019).

\section{Data and method}

\section{a. Airborne radar data}

Four P3 missions produced 14 passes with adequate data coverage through the inner core. During each pass, the TDR employed a scanning strategy that alternated between the fore and aft directions to produce pseudo-dual-Doppler measurements (Gamache et al. 1997). Passes were separated by approximately $1 \mathrm{~h}$, with the time between center fixes not exceeding $2 \mathrm{~h}$ except between missions. Thirty minutes of TDR data provided enough coverage to create an analysis of the inner core of Michael during a pass. Details of the mission durations and center fix times are given in Fig. 1a.

\section{b. Radar quality control (QC)}

Thorough QC efforts were necessary to remove non-weather echoes and prepare the raw TDR data for dual-Doppler analysis.
Navigation errors introduced by movements of the aircraft within the storm and uncertainties in the inertial navigation system were corrected to obtain more accurate Doppler velocity and pointing angles (Cai et al. 2018). Subsequently, data from each radar scan were given an initial QC effort by the algorithm developed by Bell et al. (2013) that uses the SOLO II radar editing software from the National Center for Atmospheric Research (Oye et al. 1995). The algorithm is a first step to successful removal of noise, ground clutter, second trip echoes, and other nonmeteorological data that can prevent accurate wind synthesis from TDR data. Velocity unfolding, which is necessary in a hurricane where wind speeds exceed the Nyquist velocity of the TDR, is included in the algorithm. Even after automated QC, some manual effort was required to produce an accurate dual-Doppler wind synthesis. Two missions, originating on 8 October and 10 October, received manual QC.

The time-consuming nature of manual QC created a need to expedite the process so as to analyze all available aircraft passes. A dataset composed of the postmanual QC TDR scans from the three passes during the 10 October flight was compiled to produce a "random forest" machine-learning model that was capable of recreating manual QC. The technique is fully described in DesRosiers (2020) and is briefly summarized here. After initial testing to set model parameters and improve accuracy, data from the first and third passes were used to train the model while withholding data from the second aircraft pass for subsequent evaluation. The model was tasked with performing QC on the second pass through Hurricane Michael on 10 October, which it had not seen during training. A spot check for any data that should have been removed by the model was performed to investigate how close the machine-learning QC method could mirror the already completed manual effort. Dual-Doppler analyses were produced from the data cleaned by the model, data that received a subsequent spot check, and the original manual 
effort. All three analyses were compared to determine whether the method could be used to QC data from the remaining two flights. The wind field obtained via machinelearning QC was found to be qualitatively similar to manual QC. The wind field after an additional spot check was nearly identical to manual QC. Successful completion of the test allowed for the QC of remaining TDR data from both missions on 9 October with the machine-learning model. Additional manual checks were performed on all passes that received model QC to maintain a similar standard of QC across the dataset.

\section{c. Dual-Doppler analyses}

Dual-Doppler analyses were created for each pass with Spline Analysis at Mesoscale Utilizing Radar and Aircraft Instrumentation (SAMURAI) software (Bell et al. 2012; Foerster and Bell 2017), which is part of the Lidar Radar Open Software Environment [LROSE; Bell (2019)]. The three-dimensional (3D) variational data assimilation technique uses radar observations and cubic b-spline basis functions to produce a best guess at the most likely state of the atmosphere by minimizing a cost function. SAMURAI solves for the 3D wind field using the aforementioned techniques with the addition of mass continuity as a numerical constraint. The resulting wind field is represented as a $3 \mathrm{D}$ cubic spline function that is analytically differentiable to calculate vorticity and divergence. The dual-Doppler winds were synthesized using a 1-km horizontal spline nodal spacing and 0.5$\mathrm{km}$ vertical nodal spacing with a $4 \Delta x$ Gaussian filter in the horizontal plane and $2 \Delta x$ filter in the vertical direction applied. With this spline nodal spacing and Gaussian filter length, the minimum resolved scale is $\sim 4 \mathrm{~km}$ in the horizontal direction, or approximately 2.85 times the along-track data spacing of $\sim 1.4 \mathrm{~km}$. To minimize issues from attenuation, the $2^{\circ}$ beamwidth of the $X$ band (3-cm wavelength) radar, and data coverage, the analysis was limited to the innermost $60 \mathrm{~km}$ of the storm.

Figure 2 shows reflectivity cross sections during the first and last pass of each of the four missions that illustrate the changes in radar presentation and organization of the storm's inner core as it underwent RI in the Gulf. Cross sections are shown at $6 \mathrm{~km}$ to place emphasis on the increasing organization in the midlevels of the storm. Increases with time in symmetric coverage of high reflectivity values denoting stronger convection are indicative of intensification. The increasing symmetry is important to findings of this study and will be examined in detail. However, asymmetric aspects of Hurricane Michael's evolution are also of importance and have been discussed by Cha et al. (2020).

\section{d. Thermodynamic retrieval}

A radar-based thermodynamic retrieval technique developed for use in rapidly rotating vortices (Foerster and Bell 2017; Boehm and Bell 2021) was used to discern the thermodynamic structure in the storm. The technique can retrieve perturbations in pressure and temperature relative to a horizontally varying reference state from the dual-Doppler winds. The thermodynamic retrieval derives pressure and temperature perturbations as three-dimensional functions using a similar spline-based variational method as the SAMURAI wind retrieval. In the current
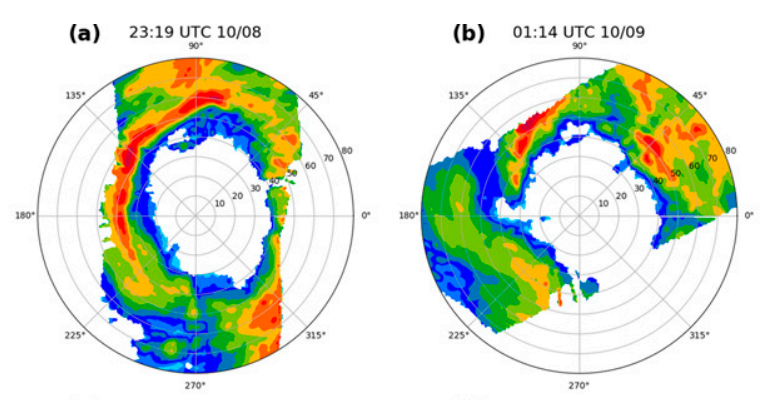

(c) $\quad 09: 12 \underset{\text { UT. }}{\text { UTC }} 10 / 09$

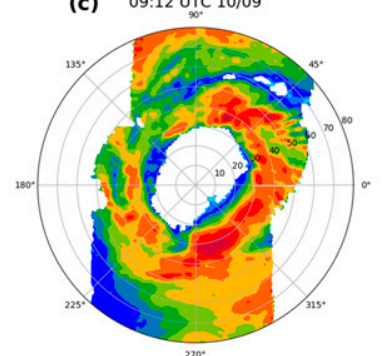

(d) 13:13 UT: $10 / 09$

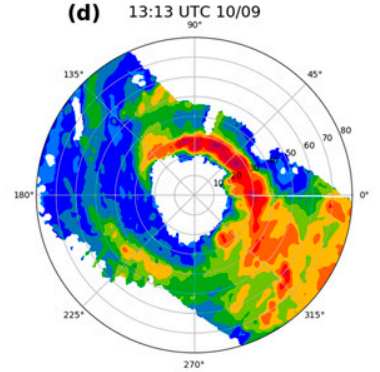

(e) 21:29 UTC 10/09

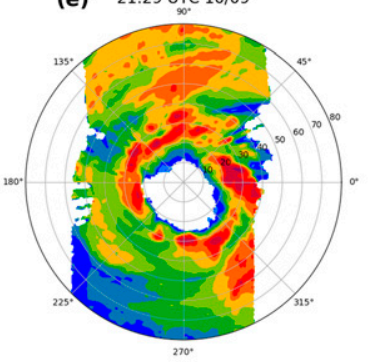

(g) 09:49 UTC $10 / 10$
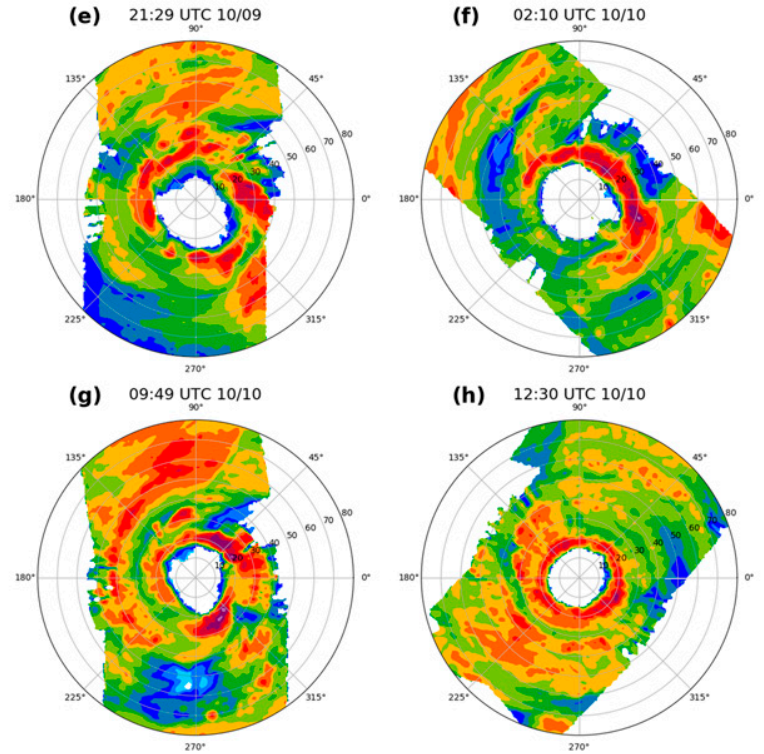

(h) $\quad 12: 30$ UTC $10 / 10$

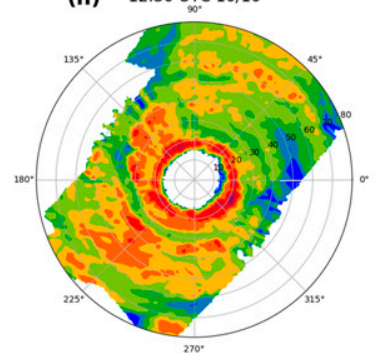

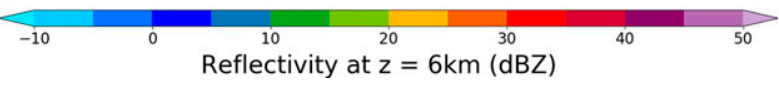

FIG. 2. Polar plots of radar reflectivity at $6-\mathrm{km}$ altitude. The first and last aircraft pass from (a),(b) 1008H1; (c),(d) 1009H1; (e),(f) $1009 \mathrm{H} 2$; and $(\mathrm{g}),(\mathrm{h}) 1010 \mathrm{H} 1$ are shown, with center fix times displayed at the top of each panel.

study we use a thermal-wind-balanced reference state that is calculated using an azimuthally averaged tangential wind field and the vertical temperature profile at a large radius. The moist tropical sounding from Dunion (2011) was used for the outer vertical temperature profile. The sounding was chosen as a time invariant climatological reference point for evaluating changes in temperature within the storm as recommended by Stern and Zhang (2016). The temperature and pressure fields of the reference state are obtained by integrating the thermal wind equation inward from the large radius boundary. For the thermal wind 

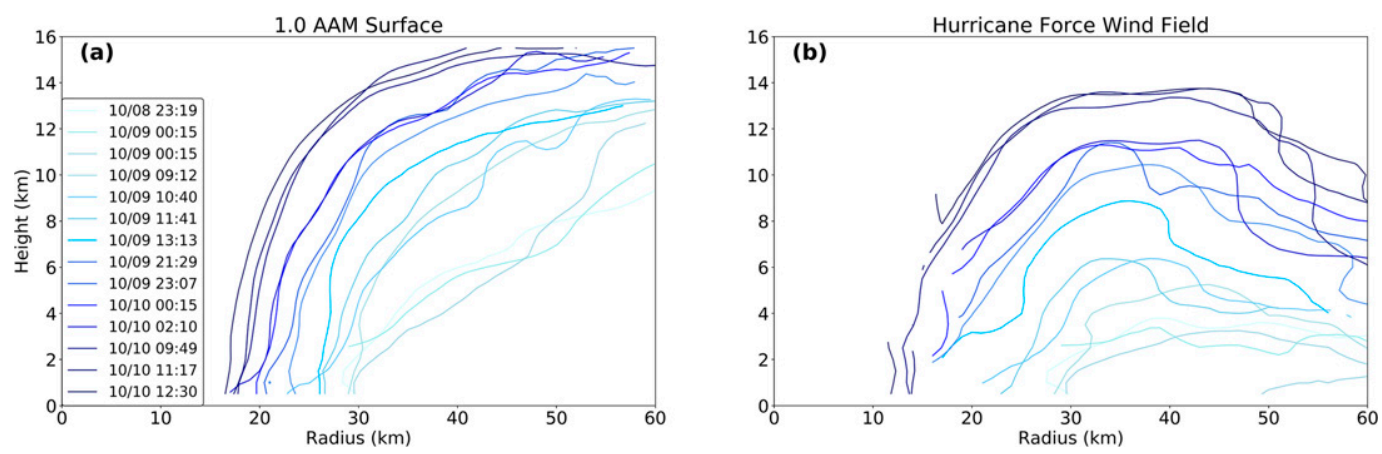

FIG. 3. (a) Progression of the azimuthally averaged $1 \times 10^{6} \mathrm{~m}^{2} \mathrm{~s}^{-1}$ absolute angular momentum (AAM) contour, and (b) hurricane-force $33 \mathrm{~m} \mathrm{~s}^{-1}$ wind contour during each of the 14 aircraft passes.

calculation, the mean tangential wind is assumed constant and unchanging from the $2-\mathrm{km}$ vertical level to the surface due to uncertainties in the applicability of gradient wind balance in the boundary layer. This assumption does not affect our conclusions because our main focus in this study is on upper levels. Following the variational retrieval, we summed the retrieved perturbations and reference state to obtain full three-dimensional fields of pressure and density potential temperature $\theta_{\rho}$. We then azimuthally average the temperature similarly to the wind field to produce the axisymmetric temperature field $\bar{\theta}_{\rho}$.

\section{e. Azimuthal mean calculation}

Axisymmetric contributions to the RI process are the primary focus of the current study. Analyses that depict the azimuthal mean quantities as functions of radius and height are used to show the evolution of Michael's structure. Each Cartesian wind analysis, performed with radial resolution of $1 \mathrm{~km}$ and vertical resolution of $0.5 \mathrm{~km}$, was regridded to a polar coordinate system consisting of radius, azimuth, and height with azimuthal resolution of $1^{\circ}$. To prevent isolated data points from biasing values, a $50 \%$ threshold was used as a minimum for calculating the azimuthal mean. The $50 \%$ threshold was chosen based on allowable gap size in observations that preserve wavenumber-0 axisymmetric structure (Lorsolo and Aksoy 2012). The gap restriction allowed for calculation of the azimuthal mean quantities out to $60 \mathrm{~km}$, with the innermost $40 \mathrm{~km}$ having the best coverage from the TDR in the azimuth dimension, as evidenced by the topdown radar reflectivity cross sections (Fig. 2). (Figures 8 and 12 are zoomed in to only $40-\mathrm{km}$ radius to enhance visual detail of the analysis in the eyewall region.)

\section{Inner-core changes throughout RI}

The availability of 14 passes allowed for detailed documentation of the inward progression of angular momentum surfaces and growth of the wind field. Absolute angular momentum (AAM) is calculated using

$$
\mathrm{AAM}=r v_{t}+\frac{1}{2} f r^{2}
$$

where $f$ is the Coriolis force, $v_{t}$ is tangential wind, and $r$ is radius from storm center. The progression of the $1.0 \times 10^{6} \mathrm{~m}^{2} \mathrm{~s}^{-1}$
AAM contour during each pass is shown in Fig. 3a. The surface moved radially inward and became increasingly vertically aligned at higher altitudes with time. The evolving tangential wind field is shown in Fig. $3 \mathrm{~b}$ by tracking the changes in the hurricane-force $\left(33 \mathrm{~m} \mathrm{~s}^{-1}\right)$ wind contour. The contour outline became broader and deeper in response to an increase in the area of hurricane-force winds in the vortex. As RI proceeded, hurricane-force winds extended farther radially outward from the center and increased in depth through the troposphere.

\section{Changes in angular momentum and vorticity}

Angular momentum and vertical vorticity changes during each of the four aircraft missions were calculated by a point subtraction method normalized to hourly tendencies by taking the difference between the center fix times of the first to last pass in hours. Changes in AAM $(\Delta M)$ are calculated by

$$
\Delta M=\left(\mathrm{AAM}_{\text {Last }}-\mathrm{AAM}_{\text {First }}\right) /\left(t_{\text {Last }}-t_{\text {First }}\right)
$$

at each radius and height between the first and last center fixes of an aircraft mission. Flights took approximately $2-5 \mathrm{~h}$ between the first and last center fix of each depending on the amount of time spent in storm by the P3 (Fig. 1a). Figure 4 shows the calculated changes in AAM in color with contours of the AAM surfaces during the final pass in each panel to illustrate the resulting structure after changes. AAM changes during mission $1008 \mathrm{H} 1$ in Fig. 4a are rather modest and not well organized with negative magnitudes observed in the eyewall. The weak AAM change is likely related to both the brief pause in intensification (Fig. 1a) and the prevalence of asymmetries at this time (Figs. 7a-c) that may not project well onto an azimuthal average. Sharp gradients in the AAM surfaces are indicative of the eyewall location. Over the following three missions, increases in $\Delta M$ concentrate mainly in the eyewall region with peak magnitudes in the midlevels during the two missions in Figs. 4b and 4c. A weaker peak positive magnitude is observed in the final mission that extends through the bulk of the eyewall region with peak hourly rates of gain in excess of $0.1 \times 10^{6} \mathrm{~m}^{2} \mathrm{~s}^{-1}$ near $8-\mathrm{km}$ altitude. Consistent positive $\Delta M$ values were observed in the upper troposphere during all missions. The $\Delta M$ values during the final three missions are mainly positive, indicating larger values of AAM 

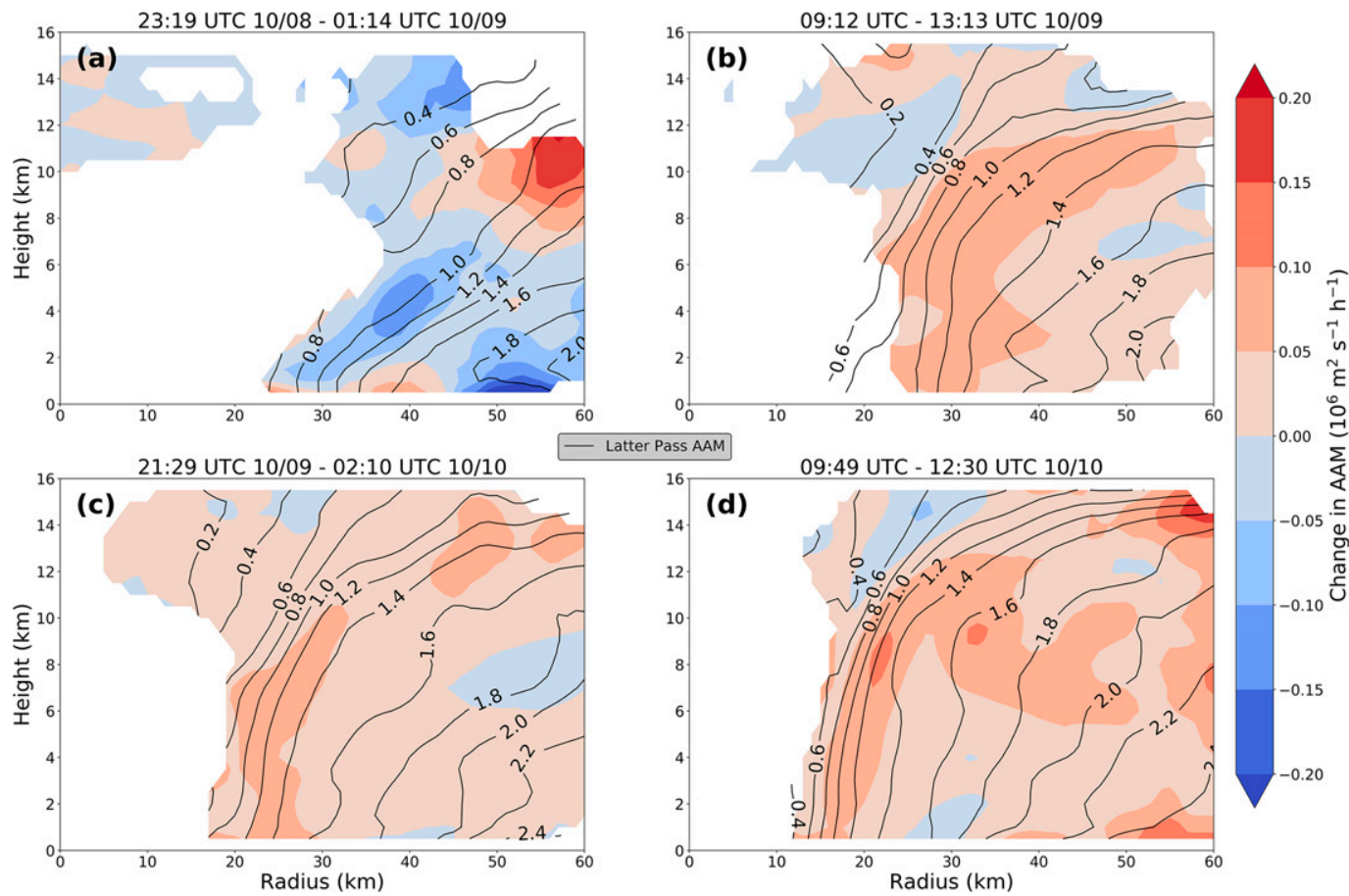

FIG. 4. The $\Delta M$ per hour shaded in color from the first to the last center fix of each aircraft mission. Differences are calculated $\left(10^{6} \mathrm{~m}^{2} \mathrm{~s}^{-1} \mathrm{~h}^{-1}\right)$ during (a) $1008 \mathrm{H} 1$, (b) $1009 \mathrm{H} 1$, (c) $1009 \mathrm{H} 2$, and (d) $1010 \mathrm{H} 1$. Positive values show increases in $M$ over time. Contours show the AAM $\left(10^{6} \mathrm{~m}^{2} \mathrm{~s}^{-1}\right)$ structure at the time of the final fix.

moving radially inward throughout the inner core of Hurricane Michael.

Vertical vorticity $\zeta$ changes are determined by the same point subtraction method used for $\Delta M$ :

$$
\Delta \zeta=\left(\zeta_{\text {Last }}-\zeta_{\text {First }}\right) /\left(t_{\text {Last }}-t_{\text {First }}\right)
$$

The magnitudes of $\Delta \zeta$ per hour, shown in color in Fig. 5, increase with time throughout intensification, with the exception of a more modest increase mostly below hourly gains of $50 \times$ $10^{-5} \mathrm{~s}^{-1}$ observed during mission $1009 \mathrm{H} 2$ (Fig. 5c). Contours of absolute vorticity observed during the last pass of each mission show the structure present after changes during each mission. The nonlinear rates with which internal organization and magnitude of vertical vorticity increase are apparent when tracking changes through each mission. The main region of positive $\Delta \zeta$ began as a broad outward-tilted area and became more focused in the eyewall region with time. Large magnitudes of positive $\Delta \zeta$ in excess of $50 \times 10^{-5} \mathrm{~s}^{-1}$ per hour first concentrate in the middle to upper levels in Fig. 5b during 1009H1. In Figs. 5c and 5d positive $\Delta \zeta$ begins to concentrate at all levels throughout the eyewall region with a slight lapse in the midlevels during mission 1009H2 (Fig. 5c). A well-defined signal for vorticity gain was present during $1010 \mathrm{H} 1$ with peak magnitudes over $100 \times 10^{-5}$ $\mathrm{s}^{-1}$ per hour in lower and upper levels (Fig. 5d). During mission $1010 \mathrm{H} 1$, a strong dipole in $\Delta \zeta$ develops with negative values appearing around $20-\mathrm{km}$ radius.
The axisymmetric tangential wind field from the last seven passes through Hurricane Michael is shown in Fig. 6, during which time a consistent vertical development of the inner core was observed. Maximum azimuthally averaged values of tangential wind increased at an approximate pace of $1 \mathrm{~m} \mathrm{~s}^{-1}$ $\mathrm{h}^{-1}$ over the course of these last two missions and increased in depth throughout the troposphere. The RMW became more vertical and contracted inward. A radially inward jump in RMW at $1-\mathrm{km}$ altitude in one pass was likely due to issues of P-3 flight path affecting radar geometry and was corrected (Fig. 6a). The expansion of tangential wind values upward during the final pass (Fig. 6g) shows that wind speeds within $6 \mathrm{~m} \mathrm{~s}^{-1}$ of the peak azimuthally averaged value $\left(66 \mathrm{~m} \mathrm{~s}^{-1}\right)$ are found up to roughly $7 \mathrm{~km}$. As the tangential wind field strengthened and grew in depth, the region where the vertical gradient was maximized became located at increasing heights in the vortex. The importance of this vertical gradient of tangential wind to the RI process is discussed in the following section.

\section{Axisymmetric vertical development of the vorticity tower}

The latter two aircraft missions into Hurricane Michael prior to landfall documented a part of the RI event characterized by consistent vertical development of the eyewall vorticity tower into the upper levels. The novel and detailed 

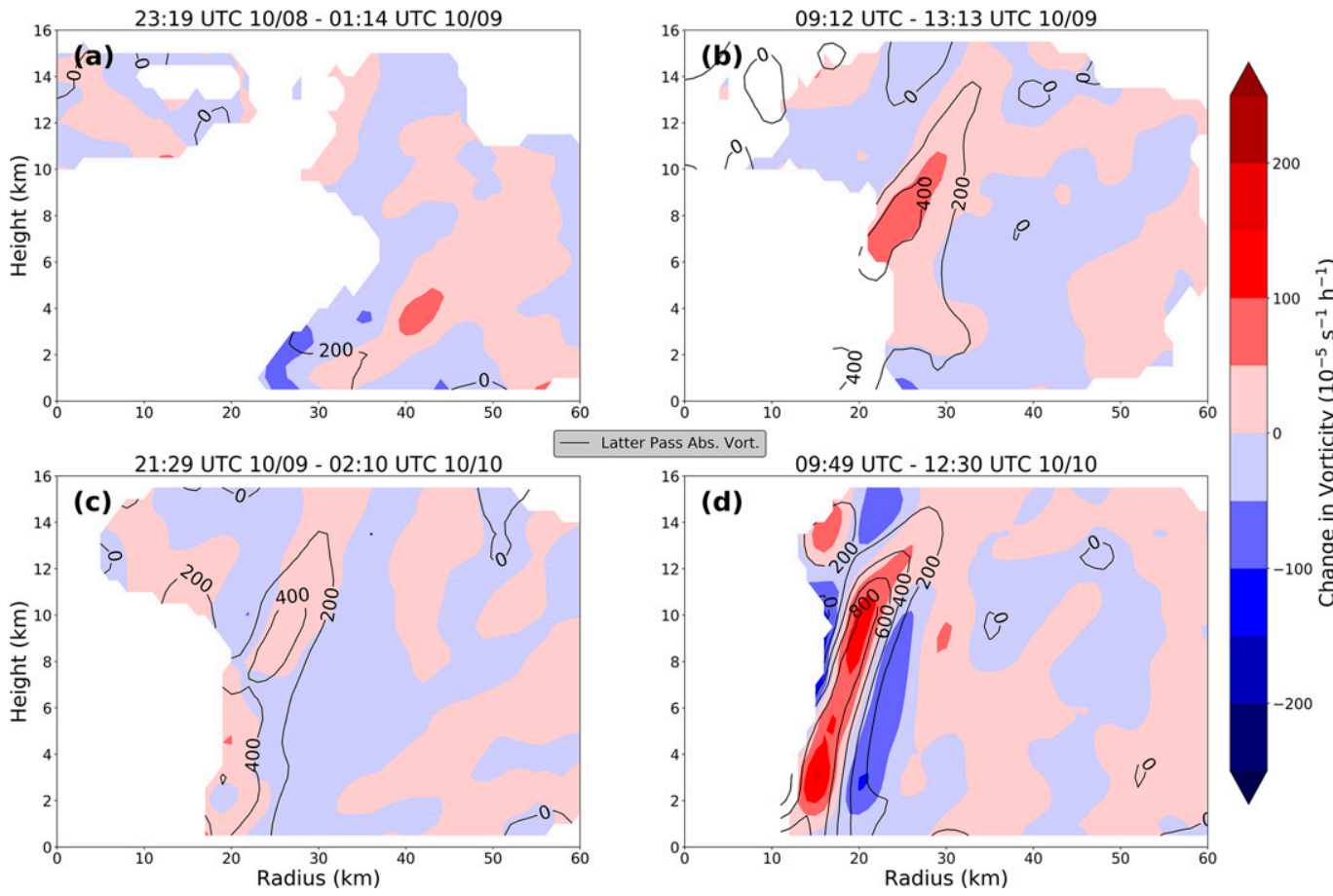

FIG. 5. The $\Delta \zeta$ per hour shaded in color from the first to the last center fix of each aircraft mission. Differences are calculated $\left(10^{-5} \mathrm{~s}^{-1} \mathrm{~h}^{-1}\right)$ during (a) 1008H1, (b) 1009H1, (c) 1009H2, and (d) 1010H1. Positive values show increases in absolute vertical vorticity over time. Contours show the vorticity $\left(10^{-5} \mathrm{~s}^{-1}\right)$ structure at the time of the final fix.

look of how RI takes place in the upper levels gives a unique opportunity to diagnose mechanisms that may have been responsible. Axisymmetric dynamics played a large role in intensification during the latter two missions and a vorticity budget approach was used to identify the increasingly axisymmetric nature of Hurricane Michael's RI. Thermodynamics were also at play in the vertical expansion taking place atop the eyewall. The impacts of both dynamics
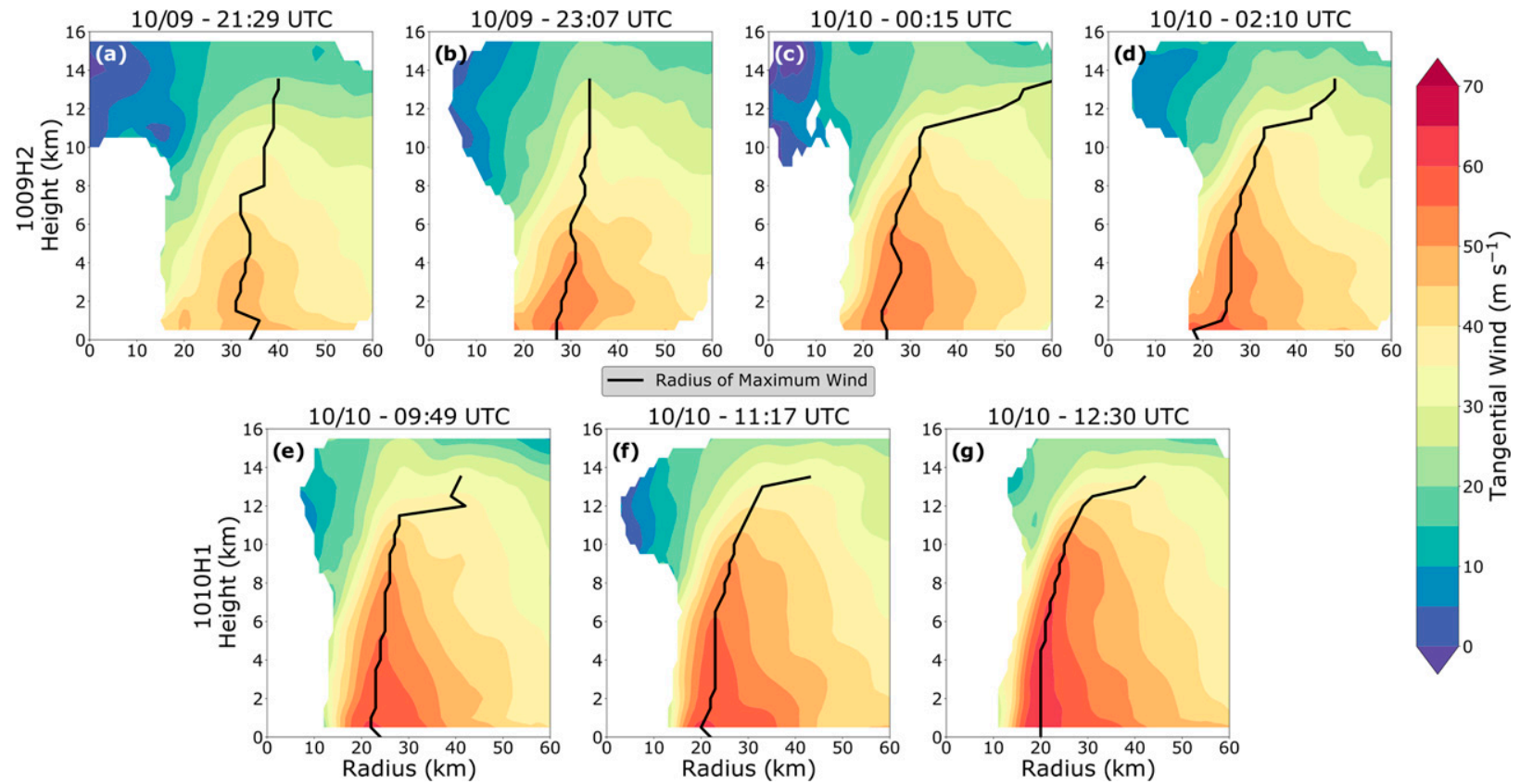

FIG. 6. Progression of the azimuthally averaged tangential wind field (colors; $\mathrm{m} \mathrm{s}^{-1}$ ) during the last two aircraft missions, (a)-(d) $1009 \mathrm{H} 2$ and (e) $-(\mathrm{g}) 1010 \mathrm{H} 1$, prior to landfall. The radius of maximum wind is denoted in black. Analysis times are listed above each panel. 
and thermodynamics on storm structure are considered to evolve together as the temporal gap between observations is too great to determine if one leads the other.

\section{a. Increasing symmetry of $R I$}

Existing TC intensification theories focus on both axisymmetric and asymmetric modes. Studies that focus on the axisymmetric mode often build on the Sawyer-Eliassen equation (Eliassen 1951) to explain symmetric intensification in a framework termed the balanced vortex model. In the model, a forced secondary circulation comprising lowerlevel inflow, updrafts and latent heating near the center, and outflow aloft becomes stronger and drives intensification (Vigh and Schubert 2009). The rotating convection paradigm seeks to explain intensification through an asymmetric process. The framework emphasizes localized rotating convective updrafts in the eyewall of a TC that enhance organization and structure of the storm's inner core (Montgomery 2016). Both theories are critical to understanding TC intensification; however, they vary on the point of symmetry in the vortex and intensification mechanism. A cylindrical coordinate vorticity budget equation (Reasor et al. 2009) was used to examine the evolution of the symmetry of intensification during Hurricane Michael's RI:

$$
\begin{aligned}
& \frac{\partial_{L}}{\partial t} \bar{\zeta}=-\bar{u} \frac{\partial \bar{\zeta}}{\partial r}-\bar{w} \frac{\partial \bar{\zeta}}{\partial z}-\left(f_{0}+\bar{\zeta}\right) \bar{\delta}-\frac{\partial \bar{w}}{\partial r} \frac{\partial \bar{v}}{\partial z} \\
& -\left[\overline{u^{\prime} \frac{\partial \zeta^{\prime}}{\partial r}}+\frac{\overline{v^{\prime}} \frac{\partial \zeta^{\prime}}{r}}{\partial \lambda}\right]-\overline{w^{\prime} \frac{\partial \zeta^{\prime}}{\partial z}}-\overline{\zeta^{\prime} \delta^{\prime}}-\left[\overline{\frac{\partial w^{\prime}}{\partial r} \frac{\partial v^{\prime}}{\partial z}}+\frac{1}{r} \frac{\partial w^{\prime}}{\partial \lambda} \frac{\partial u^{\prime}}{\partial z}\right]
\end{aligned}
$$

where prime terms denote eddy, or asymmetric, contributions that vary azimuthally, an overbar indicates an azimuthal average of the quantity, $\partial_{L} / \partial t$ is the local time tendency, $\delta$ is divergence, $f_{0}$ is the Coriolis parameter, and $\lambda$ indicates the azimuthal dimension in cylindrical coordinates. Confidence in the exact partitioning of vorticity tendency between the axisymmetric and asymmetric components is lowered by the temporal resolution of the analyses, but the budget approach is useful for examining changes in symmetry throughout the whole of the observed RI event. The first line on the righthand side of Eq. (4) is composed of four terms that quantify the vertical vorticity tendencies associated with 1) axisymmetric radial advection, 2) vertical advection, 3) stretching, and 4) tilting of horizontal vorticity. A summation of these first four terms gives a total axisymmetric vorticity tendency at the time of an aircraft pass. The second line of the rhs of the equation is made up of four terms (5-8) corresponding to the same aforementioned tendencies but instead as asymmetric eddy contributions. Terms 5 and 8 (in square brackets) are composed of two expressions. Summing these second-line terms yields a total asymmetric vorticity tendency. A sum of all terms in the budget produces a total vorticity tendency for both symmetric and asymmetric contributions at the time of the analysis.

Figure 7 shows the three summation values of asymmetric, axisymmetric, and total tendencies. Confidence in tendency values within the eye at smaller radii is low due to potential numerical instability as radius goes to zero in the calculation of Eq. (4). A single pass from each mission was chosen to illustrate the RI process as it proceeded from a mainly asymmetric to mainly axisymmetric mode. The increased symmetry is most evident in the passes from the first and last missions. Figures $7 \mathrm{a}$ and $7 \mathrm{c}$ are nearly identical as asymmetric vorticity tendency terms dominated the changes occurring on 8 October. Figures $7 \mathrm{k}$ and 71 are nearly identical on 10 October when axisymmetric tendency terms dominated. The increasing axisymmetry and decreasing asymmetry with time is further confirmed by a Pearson correlation calculation for each pass shown in Fig. 7. The variance explained $r^{2}$ by the asymmetric and axisymmetric tendencies to the total tendency shows that asymmetric intensification was more dominant early on but axisymmetric intensification became a more appropriate conceptual model in the later stages of RI. These results are consistent with tangential wind momentum budget analysis of modeled fields of Hurricane Michael where mean transport terms dominated the net tangential wind spinup in later RI as compared with eddy terms in early RI (Green et al. 2021). In the following section, we focus on the latter period where axisymmetric dynamics dominate so as to further investigate the growth of the vorticity tower during RI.

\section{b. Dynamical impacts}

Persistent spatial patterns were identified in the vorticity budget tendencies during the latter half of Michael's observed RI. Here we focus on 1230 UTC 10 October when the axisymmetric tendencies were greatest prior to landfall. Figure 8 shows the calculated axisymmetric vorticity tendency terms from the final aircraft pass, with contours overlaid showing the total summation of all tendency terms in the budget including asymmetries. As shown in Figs. 7j-1, the total vorticity tendency observed was dominated by axisymmetric terms at this time. The radial advection (Fig. 8a) and vertical advection (Fig. 8b) have oppositely signed, sloped tripole patterns maximized in the upper troposphere. Similarly, stretching (Fig. 8c) and tilting (Fig. 8d) largely oppose each other in an upper-tropospheric tripole pattern, with the negative stretching tendency having a larger magnitude in the lower troposphere. Qualitatively, the radial advection and stretching tendencies have similar spatial patterns, and their sum can be considered as the radial flux divergence of vorticity. Both the vertical advection and tilting tendencies also have similarities, and their sum can be considered as the vertical flux divergence of vorticity. While all four terms are important, we argue here that the vertical development of the tower into the upper levels of the troposphere was accomplished through reorientation of horizontal vorticity into the vertical and the transport of existing vertical vorticity upward by the positive vertical velocity in the eyewall. The strong positive tilting tendency on the inner edge of the eyewall is associated with the interaction of the vertical gradient of tangential wind and radial gradient of vertical velocity. However, only a small portion of the large tilting tendency was located in a net positive total tendency region, because much of it was offset by negative stretching tendency associated with upper-level 

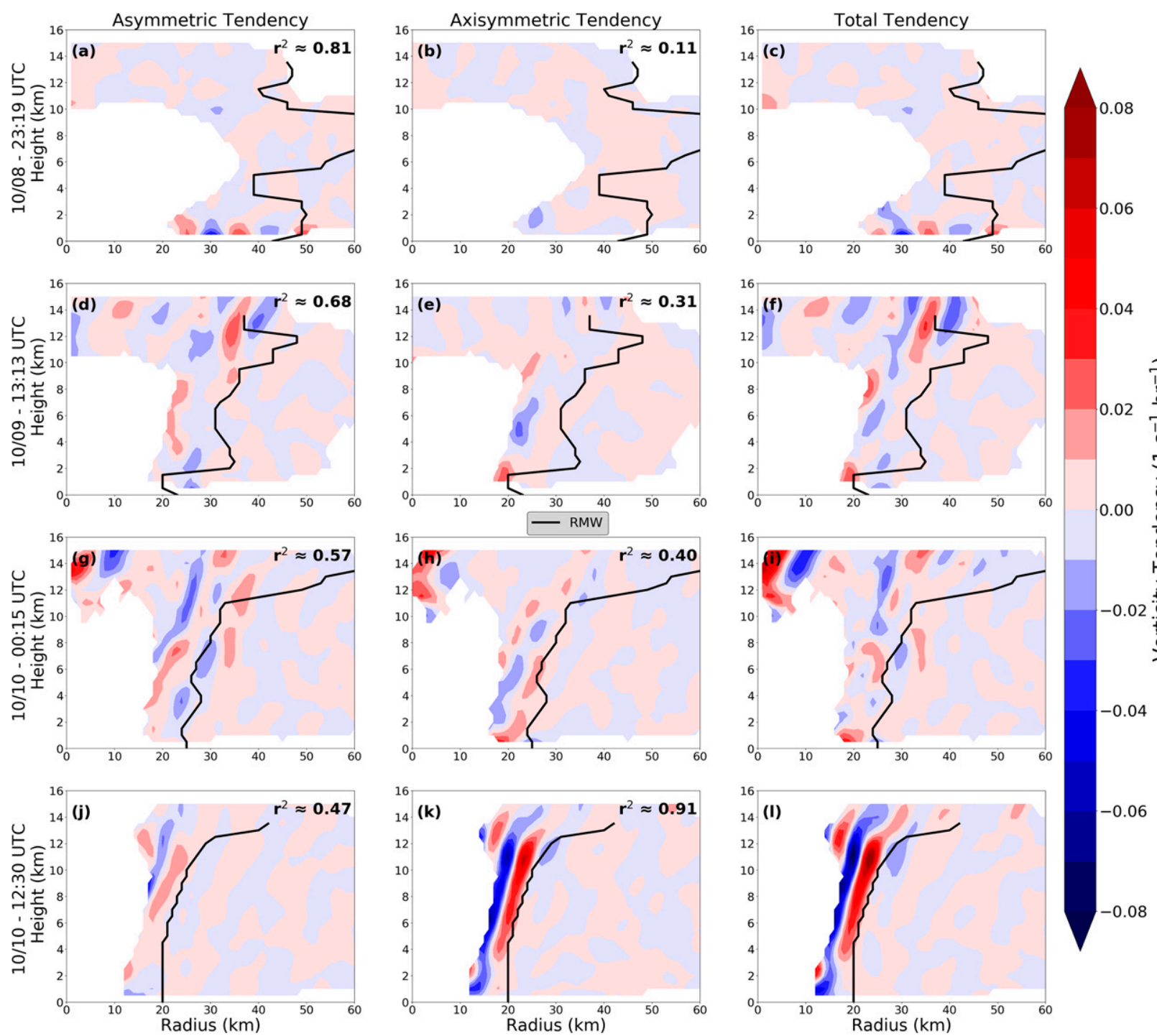

FIG. 7. Vorticity budget tendency terms from the (left) asymmetric and (center) symmetric contributions to Hurricane Michael's (right) total vorticity tendency over time. The four passes are approximately $12 \mathrm{~h}$ apart, with center fix times shown to the left of each row. A representative pass was chosen from the (a)-(c) $1008 \mathrm{H} 1$, (d)-(f) $1009 \mathrm{H} 1$, (g)-(i) $1009 \mathrm{H} 2$, and (j)-(l) $1010 \mathrm{H} 1$ aircraft missions. The radius of maximum wind is shown in black; $r^{2}$ of the total tendency by the asymmetric and axisymmetric tendency is shown in the top right of the panels.

divergence. While the tilting and vertical advection are positively associated with the updraft and upward development, we interpret the stretching and radial advection as acting together to primarily build the tower outward in the upper levels. The eyewall slope, which is greater in the upper than lower levels, is expected as based on the majority of cases analyzed sharing this characteristic in observations using airborne radar in Atlantic TCs (Hazelton and Hart 2013). The tripole pattern acts to redistribute vorticity radially inward and outward, but the magnitude of the positive tendencies is much weaker in the eye. The primary effect of the combined stretching and radial advection in the upper levels of the vorticity tower is therefore a dipole tendency that moves vorticity radially outward toward the RMW.
The net effect of all tendency terms shown creates a positive total tendency (contoured) slightly radially inward of the RMW.

To investigate the upward development of the vorticity tower, Fig. 9 shows the azimuthally averaged evolution of the horizontal and vertical vorticity over four aircraft passes from $1009 \mathrm{H} 2$ when the contribution that tilting made to storm development first became clear. In a warm-cored TC, tangential wind decays with height, which generates horizontal vorticity, mathematically defined as the negative of the vertical gradient of the tangential wind component, or $-\partial v / \partial z$. The intersection of the axisymmetric updraft, shown in the secondary circulation vectors in the top row of Fig. 9, along with the 

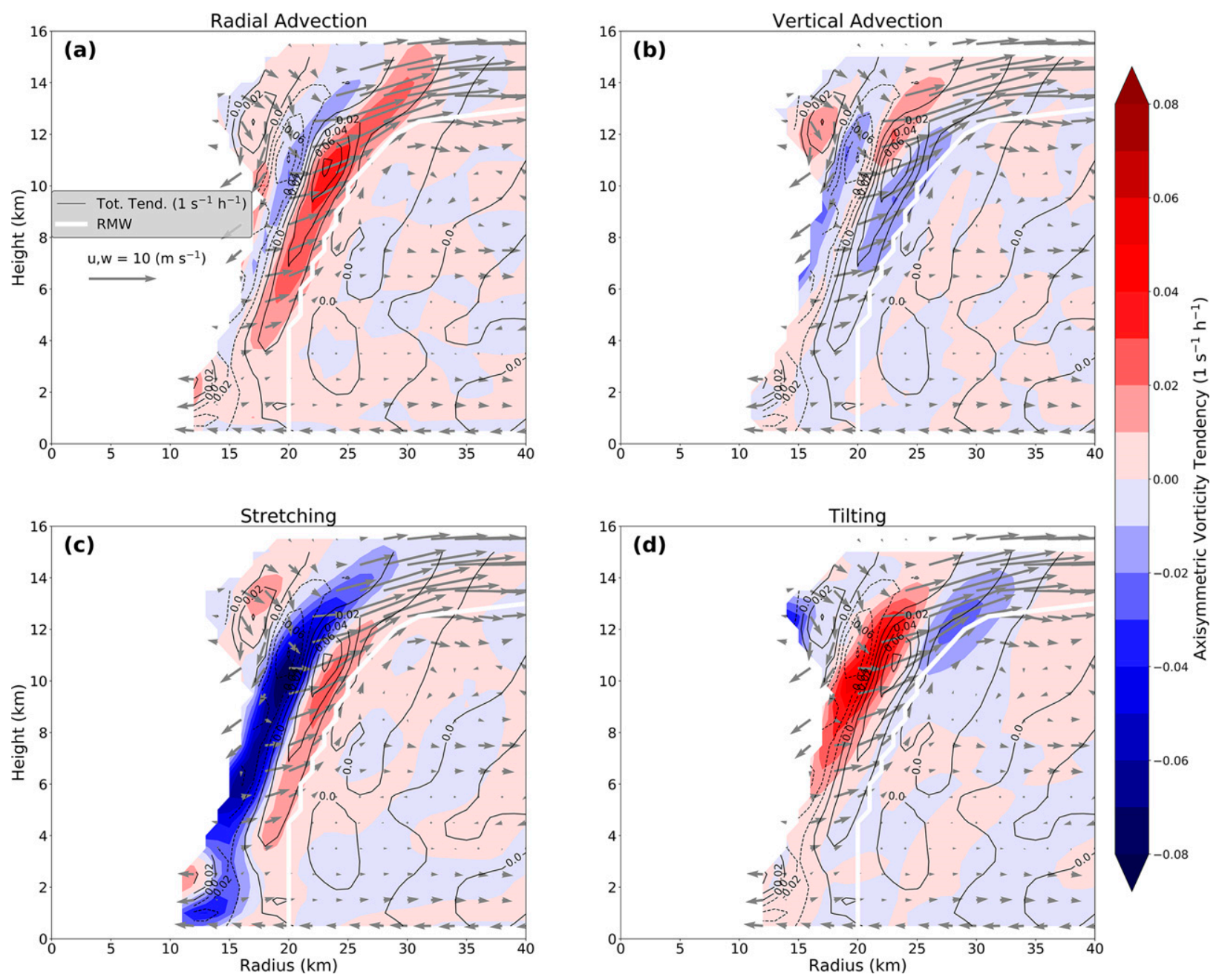

FIG. 8. Axisymmetric vorticity budget tendency terms for (a) radial advection, (b) vertical advection, (c) stretching, and (d) tilting during the final pass through Hurricane Michael prior to landfall at 1230 UTC 10 Oct 2018. The summation of all instantaneous tendency terms including asymmetric terms is contoured (also shown in Fig. 71). The radius of maximum wind is denoted in white. Secondary circulation vectors are given in gray.

horizontal vorticity shown in color, created axisymmetric tilting tendency terms in the vorticity budget [Eq. (4)]. The tendency was positive on the radially inward edge of the updraft and negative on the outer edge. The regions of positive tendency, shown in warm colors in the middle row, are areas of the storm where the horizontal vorticity was reoriented into the vertical vorticity with cyclonic rotation. There is also an accompanying negative dipole that produces vertical vorticity with anticyclonic rotation. The dipole structure between the two tendencies became more noticeable during the latter passes of $1009 \mathrm{H} 2$ in Figs. 9g and 9h. Vertical vorticity in the eyewall (Figs. 9i-1) showed increased magnitude where the positive tilting tendencies occur progressively higher in the storm. The same behavior continued with greater magnitude during the flight into Michael on 10 October prior to landfall (Fig. 10). Figure 10i shows the final observed axisymmetric vorticity tower prior to landfall exhibiting coherent and nearly uniform structure from the surface to approximately $12 \mathrm{~km}$.

\section{c. Thermodynamical impacts}

In addition to dynamical impacts, we seek to investigate the role of thermodynamic factors in Hurricane Michael's RI using the observations. The radar-based thermodynamic retrieval technique described in section $3 \mathrm{~d}$ (Foerster and Bell 2017) was applied to the winds during the final two aircraft missions $(1009 \mathrm{H} 2$ and $1010 \mathrm{H} 1)$. Here, we focus on the retrieved density potential temperature to assess the evolution of the TC warm core.

The dynamics and thermodynamics are closely coupled via thermal wind balance as described by the following relationship:

$$
\hat{f} \frac{\partial v}{\partial \tilde{z}}=\frac{g}{\theta_{\rho, 0}}\left(\frac{\partial \theta_{\rho}}{\partial r}\right)_{\tilde{z}} .
$$

Equation (5) is in pseudo-height coordinates (Hoskins and Bretherton 1972; Schubert and Hack 1982; Schubert et al. 2017) where $\hat{f}=f+(2 v / r), \tilde{z}$ is pseudo-height, $g$ is gravity, and 

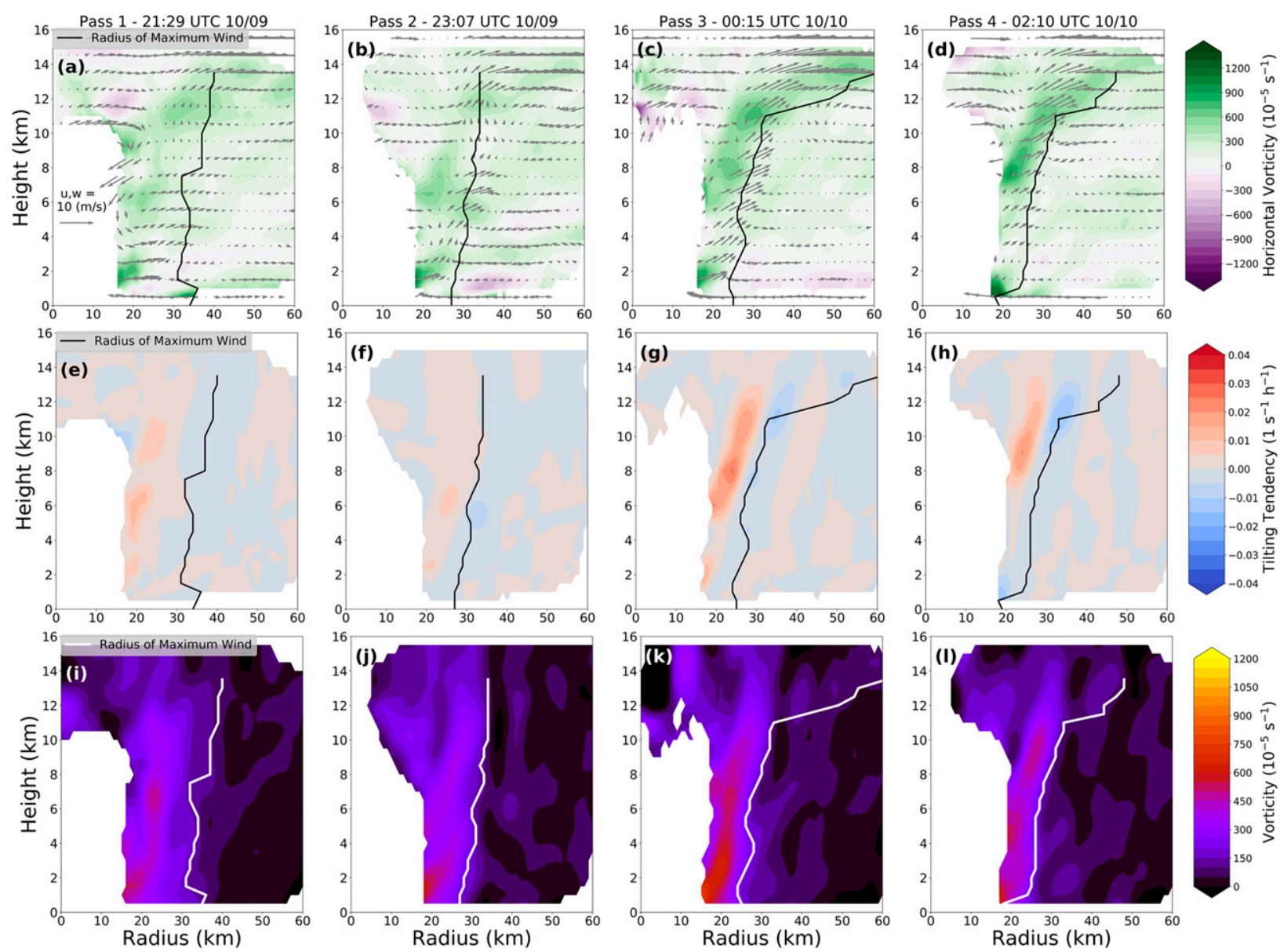

FIG. 9. Azimuthally averaged horizontal vorticity (colors; $10^{-5} \mathrm{~s}^{-1}$ ) with (top) secondary circulation vectors, (middle) axisymmetric tilting tendency of vorticity $\left(\mathrm{s}^{-1} \mathrm{~h}^{-1}\right)$, and (bottom) vertical vorticity $\left(10^{-5} \mathrm{~s}^{-1}\right)$. Analysis is from aircraft passes at (a),(e),(i) 2129 and (b),(f),(j) 2307 UTC 9 Oct and (c),(g),(k) 0015 and (d),(h),(l) 0210 UTC 10 Oct during aircraft mission $1009 \mathrm{H} 2$.

$\theta_{\rho, 0}$ is a reference density potential temperature. We show the pseudo-height relationship here since it directly illustrates the specific thermodynamic quantity retrieved in our analysis $\bar{\theta}_{\rho}$. We note that the retrieved temperature from the observations shown here is the sum of retrieved perturbations and the reference state, and therefore does not explicitly assume thermal wind balance holds. Thermodynamic fields shown were not interpolated from height to pseudo-height coordinates for a direct assessment of thermal-wind balance, but rather we use the above equation to gain insight into the mechanisms by which dynamic and thermodynamic mechanisms work together during RI.

During $1009 \mathrm{H} 2$ and $1010 \mathrm{H} 1$, negative vertical gradients of tangential wind [left side of Eq. (5)] were present in the upper levels of the eyewall, shown as positive horizontal vorticity in Figs. 9 and 10. If the vortex is in approximate thermal wind balance, the areas with positive horizontal vorticity should be associated with negative radial gradients of density potential temperature [right side of Eq. (5)]. Figure 11 shows the retrieved density potential temperature and its radial gradient during the last two missions. The dynamics of these last two missions were analyzed in Figs. 6, 9, and 10. The lowest $2 \mathrm{~km}$ was excluded from this analysis given the aforementioned constraints of the thermodynamic retrieval method in lowest levels of the storm. The presence of the warm core was assessed by taking the $\bar{\theta}_{\rho}$ field in the inner core and subtracting the averaged profile at $r=60 \mathrm{~km}$. The warm core temperature anomaly increased in magnitude in the upper levels with time, consistent with the increasing intensity and decreasing surface pressure. Increases in $\partial \theta_{\rho} / \partial t$ shown as contours in Fig. 11 were collocated with areas of stronger horizontal vorticity during the later stages of intensification (cf. Figs. 9 and 10). Increasing warm core temperature anomalies and areas where tilting was maximized were coincident with respect to height, providing a link between dynamic and thermodynamic impacts via the thermal wind equation. Small biases in warm core temperature anomaly arise from the choice of sounding reference profile (not shown), but the conclusions are not sensitive to the sounding used in the retrieval. 

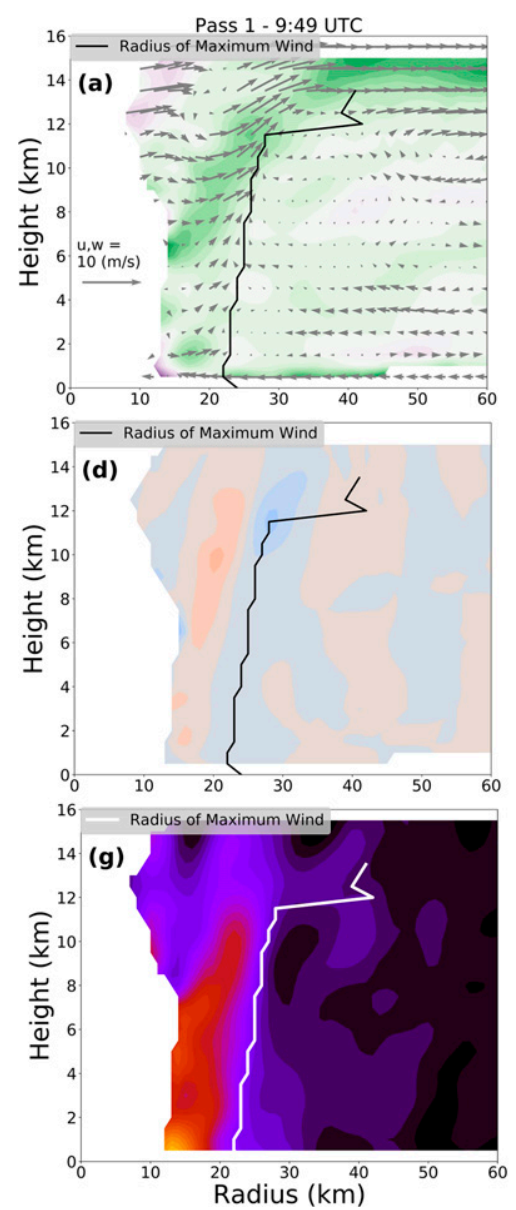
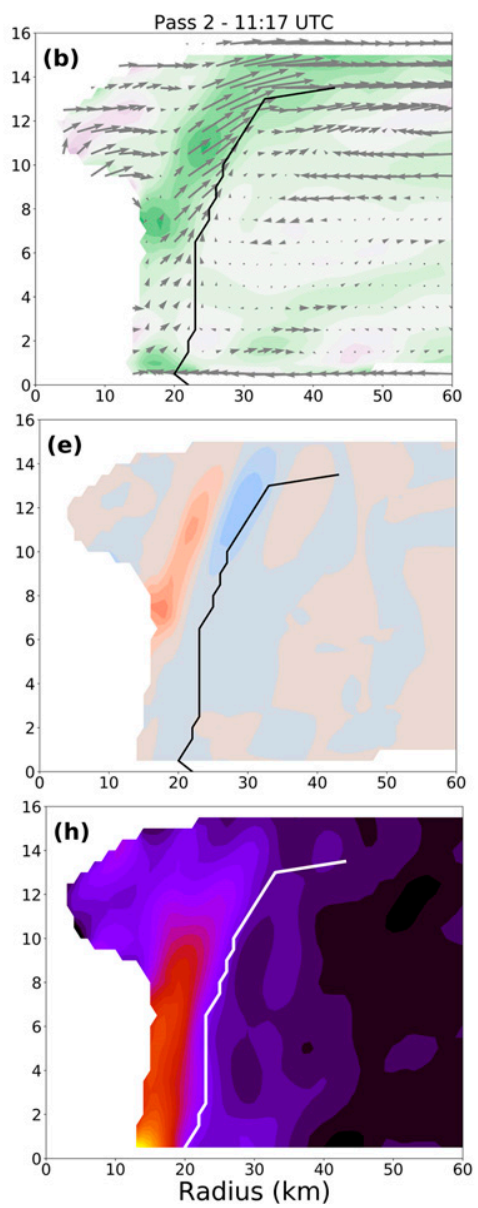
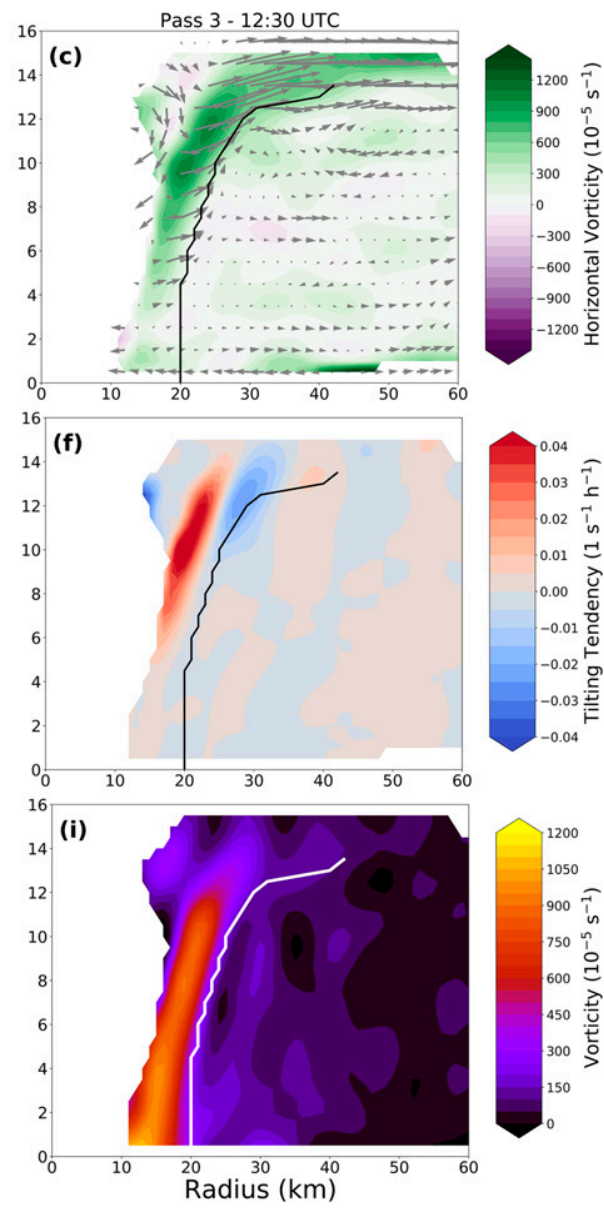

FIG. 10. As in Fig. 9, but from aircraft passes at (a),(d),(g) 0949 UTC; (b),(e),(h) 1117 UTC; and (c),(f),(i) 1230 UTC 10 Oct during aircraft mission $1010 \mathrm{H} 1$.

The cumulative picture gleaned from the analyses is that the storm continuously tilted horizontal vorticity into the vertical at increasing heights via the eyewall updraft, coupled simultaneously with the maintenance of thermal wind balance and changes to the radial gradient of temperature and an increasing warm core temperature anomaly. The collocation of tilting tendencies and the increasingly negative gradient of $\bar{\theta}_{\rho}$ provides observational evidence of the interconnected nature of the dynamic and thermodynamic processes that contributed to the vertical growth of the vorticity tower in Hurricane Michael.

\section{Discussion}

The intensification of a TC via tilting of horizontal vorticity has been examined in other contexts. In cyclogenesis, convective bursts tilt ambient low-level horizontal vorticity into the vertical while also stretching the vertical vorticity due to lowlevel convergence (Montgomery et al. 2006). In contrast to that conceptual model, the upper-level process of tilting and stretching in Michael's RI was due to axisymmetric features that allowed tilting to be a persistent upper-level process rather than an episodic low-level one. Additionally, the advection and stretching terms take on a different role here than in the genesis stage, as is discussed further below.

Some similarities to the conceptual model described here can be seen in the secondary eyewall development of Hurricane Rita (2005), where a positive vorticity tilting tendency occurred radially inward of the secondary eyewall (Didlake and Houze 2011). Structure differentiates the Rita case from the one analyzed in Michael. The reflectivity and associated convection of the forming secondary eyewall in Rita was less uniform by visual inspection than the axisymmetric convective ring observed in the mature inner eyewall of Hurricane Michael. Another difference appears to be that the updraft in the secondary eyewall of Rita was centered in the reflectivity tower, while the updraft in Michael's eyewall was displaced slightly radially outward of the center of the reflectivity tower in the sloped eyewall. This difference results in collocation of the positive tilting tendency with the top of the existing vorticity tower. We hypothesize that the specific location of the eyewall updraft and slope of both the updraft and vorticity tower played an important role in Michael's RI. Further research is needed to determine whether this hypothesis can generalize to other RI cases. 

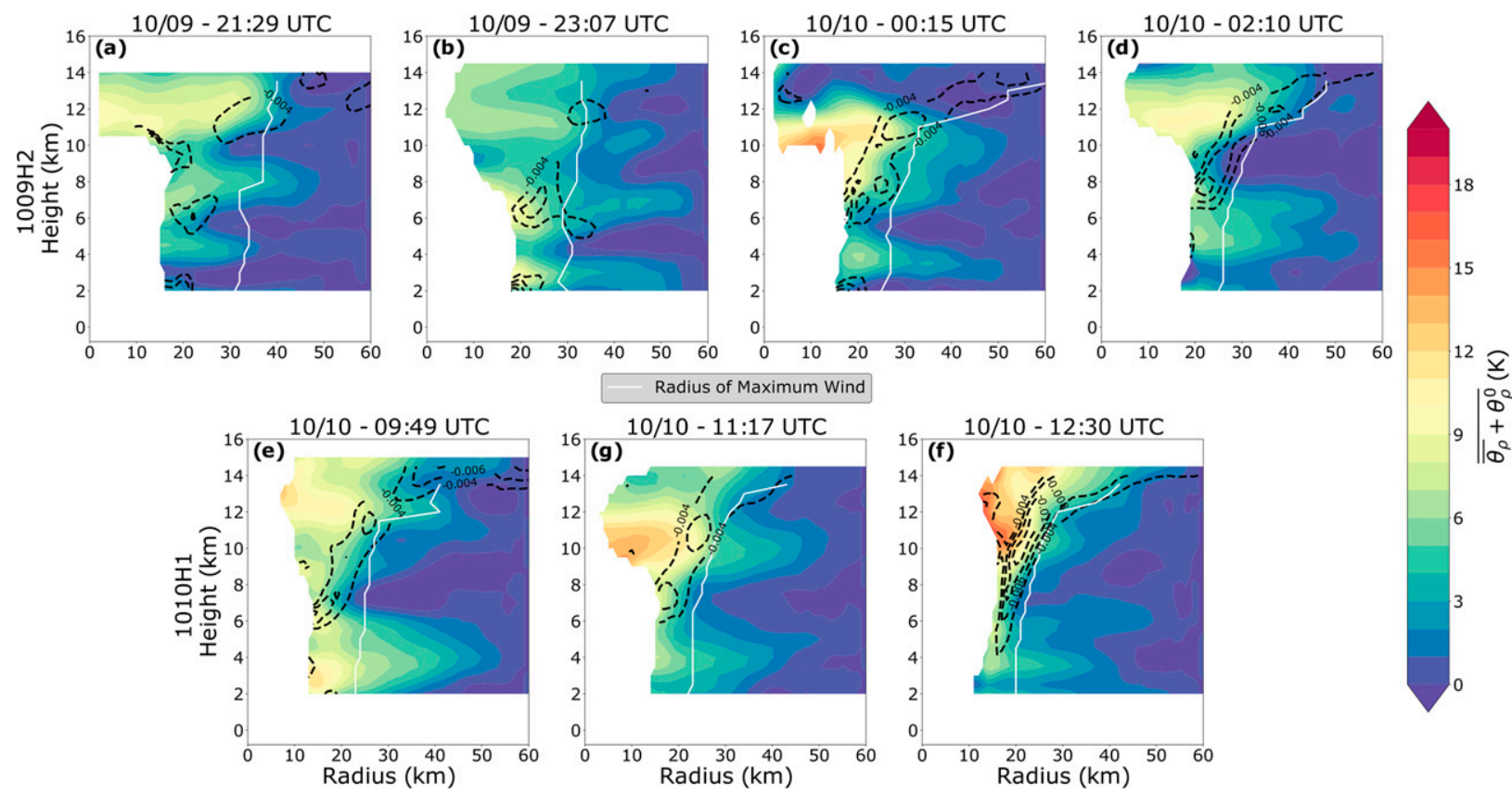

FIG. 11. Retrieved azimuthally averaged density potential temperature anomalies (colors; K) during the last two aircraft missions, (a)-(d) $1009 \mathrm{H} 2$ and (e)-(g) $1010 \mathrm{H} 1$, prior to landfall. Anomalies are calculated with respect to the retrieved vertical profile at $r=60 \mathrm{~km}$. Contours show the radial gradient of density potential temperature $\left(\mathrm{K} \mathrm{km}^{-1}\right)$. Analysis times are given above each panel.

While we have emphasized tilting herein, it was only part of the observed inner-core dynamics. The dipole nature of tilting prevents the term from increasing the net circulation on its own. The negative tilting portion of the dipole is located near strong upper-level outflow shown by secondary circulation vectors (Fig. 9d) that can advect the negative vorticity out of the inner core. The positive tilting tendency is on the inner edge of the updraft where vertical advection can transport the reorientated horizontal vorticity and preexisting vertical vorticity upward to build the tower in the vertical. Radial advection and stretching were shown to further redistribute and concentrate the vorticity outward along the sloped eyewall toward the RMW where the vorticity tower is maximized and reinforced.

The total positive vorticity tendency resulting from all axisymmetric terms was located along the inner edge of the RMW where convective heating, evidenced by the updraft, took place. Convective heating in the eyewall causes thermally indirect subsidence warming inside the eye (Willoughby 1998). Toward the end of the observation period of Michael, accompanying downdrafts on the inner edge of the upper eyewall, shown by secondary circulation vectors (Figs. 9 and 10), were observed indicators of subsidence. Resulting forced dry descent as a consequence of heating in the eyewall aids in making the eye cloud free (Houze 2010), which was observed via satellite imagery (not shown) showing a clearing eye during the final two missions. The descent leads to continued strengthening of the warm core. Additionally, diabatic heating from eyewall convection itself will also contribute to the warm core, with increasing efficiency of heating as a vortex gets stronger (Schubert and Hack 1982; Nolan et al. 2007).
Finer points of structure, location, and intensity of the warm core could not be speculated on without more concrete thermodynamic observations available above aircraft reconnaissance flight level. However, a growing warm core temperature perturbation was consistent with ongoing dynamic processes via thermal wind balance where tilting and the radial gradient of $\bar{\theta}_{\rho}$ were maximized in the upper levels. This warming in the upper levels must impact near-surface intensification of the vortex through hydrostatic balance. In the stratospheric level of insignificant dynamics (LID) assumption, which is valid on TC scales, local temperature changes at any level above the surface and below the LID contribute to the pressure tendency at the surface (Hirschberg and Fritsch 1993). Furthermore, warming in the upper levels where density is lower and there is greater spacing between isobars has a greater impact on the surface pressure than warming at lower levels would. Lower surface pressure correlates with intensification of the wind field in the lower troposphere due to a greater pressure gradient force.

Although the axisymmetric vorticity tower became more thinly concentrated in physical space, the angular momentum surfaces moved radially inward faster than the convective heating and the updraft. A comparison of the tracked AAM surface in Fig. 3a with the secondary circulation vectors (Figs. 9 and 10) supports the inward movement of angular momentum surfaces outpacing the heating. As a result, the dynamic size of heating increased along with the efficiency of heating when considered in the potential radius framework pioneered by Schubert and Hack (1983). As the eyewall remained fixed to the inside edge of the RMW, it still moved inward toward the storm center as the RMW contracted 
(Fig. 7), a behavior also linked to increased subsidence in the eye (Willoughby 1998). While we have focused here on vorticity and its tendencies from tilting, stretching, and advection, the relationship between heating and intensification is more succinct in a potential vorticity framework. Further analysis of Michael's RI from a complementary potential vorticity perspective (Martinez et al. 2019) is warranted.

Last, we note that tendency terms in the vorticity budget indicated intensification was mainly axisymmetric in the later stages of RI, but eddy contributions were nonnegligible. The generation of polygonal eyewalls and eye-eyewall mixing in TCs has been long established in theoretical frameworks and observation (Kossin and Eastin 2001; Schubert et al. 1999). More recent work simulating RI in Typhoon Megi (2010) credit mesovortices at polygonal eyewall vertices as agents of intensification via localized increases in radial AAM advection and heat fluxes within the RMW (Lee and Wu 2018). Polygonal eyewall structure was observed in Hurricane Michael as it approached the Florida coast (Cha et al. 2020), but analysis of these features and their role is beyond the scope of the current study.

\section{Conclusions}

Four missions flown by the NOAA Hurricane Hunters provided an opportunity to investigate Hurricane Michael's historic RI event in the Gulf of Mexico that led to the fourth recorded landfall of a category- 5 hurricane in the United States. Fourteen aircraft passes through the storm allowed for snapshots of the evolution in the inner core of the storm. Point subtractions between analyses at the start and end of each aircraft mission quantified changes in vertical vorticity and angular momentum. Changes in both quantities exhibited increasingly positive magnitudes that concentrated in the eyewall region as the RI event progressed. Angular momentum changes were largely positive while the vorticity changes progressed toward a dipole with positive magnitude changes in the vorticity tower and negatives just radially outside. Inward movement of angular momentum surfaces and growth of the hurricane-force wind field were documented. An analysis of the contributions of asymmetric and symmetric vorticity tendencies show that the vortex intensification progressed toward axisymmetry with greater efficiency over time.

Temporal coverage of Hurricane Michael during RI allowed for observation of the vortex as it built vertically into the upper levels of the troposphere. The mechanisms that led to this vertical development discussed in this study are illustrated schematically in Fig. 12. Tilting of the ambient horizontal vorticity, created by the decay of the tangential wind field with height, by the eyewall updraft was found to create a dipole tendency with the positive tendency inward atop the existing vorticity tower and the negative tendency radially outward of the updraft in a region of outflow. Tilting has been identified as an intensification mechanism in other stages of the TC life cycle, but the detailed observations of this process atop the eyewall of a rapidly intensifying Atlantic hurricane provide a novel look at its importance at upper levels during RI. Tilting alone cannot increase the overall circulation within the inner core, and the growth of the vortex tower required contributions from advection and stretching as

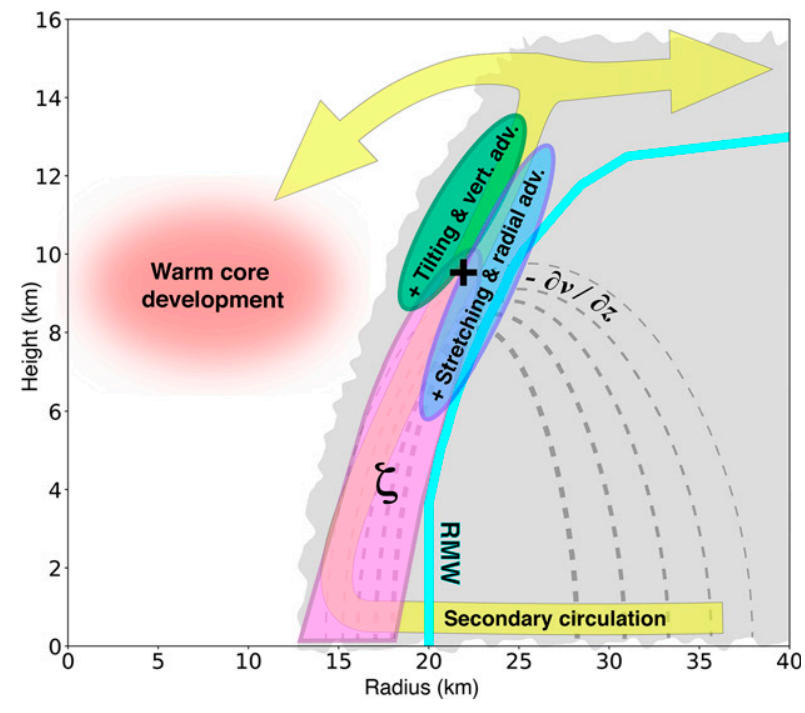

FIG. 12. Schematic representation of observed vortex development processes during rapid intensification of Hurricane Michael (2018). Light-gray shading denotes the eyewall cloud. The purpleshaded region represents the tower of high vorticity in the eyewall. Green shading denotes the region of positive vorticity tendency from tilting and vertical advection. Blue shading represents the region of positive vorticity tendency from stretching and radial advection. The plus sign denotes the location of the maximized positive net vertical vorticity tendency from the sum of these terms and negative tendencies (not shown). Yellow shading denotes secondary circulation. The cyan contour denotes the radius of maximum wind. Dashed gray contours denote tangential wind, with decreasing line thickness indicating weaker winds. The region of enhanced horizontal vorticity is denoted near 9-km altitude at the same level as the red-shaded area where warm core development is maximized through thermal wind balance.

well. Vertical advection transported vertical vorticity from the tower upward, including that reoriented by tilting, while stretching and radial advection acted to move the vorticity outward and amplify the vorticity along the inner edge of the RMW.

A thermodynamic retrieval technique provided evidence of the maintenance of thermal wind balance and an increasing warm core temperature perturbation in the upper levels where heating can have an increased impact on surface pressure. The collocation of the radial gradient of density potential temperature and tilting tendency show the interconnected nature of the dynamic and thermodynamic mechanisms of intensification. Warming in the upper levels has a more substantial impact than warming closer to the surface on lowering the minimum sea level pressure. Connections between tilting, diabatic heating, and eye subsidence that enhance the warm core can therefore lower surface pressure, allowing mechanisms of RI taking place in the upper levels to play a role in the intensification of the near-surface wind field. Hurricane Michael's well-defined axisymmetric structure shown in these analyses suggest the mechanisms explored in this study took place in a nearly continuous and efficient manner. The collocation of the sloped eyewall updraft, vertical vorticity tower, and horizontal vorticity made available for tilting by the decaying winds aloft 
were able to produce rapid surface pressure falls in Hurricane Michael. However, it is still unclear if this specific arrangement of heating and structure is a universal pathway to vertical development in RI cases. It is evident from the analyses herein that producing a stronger TC requires producing a deeper vortex. Further investigation of the intensification mechanisms presented here through both future observations and modeling studies is recommended. Improved understanding of inner-core processes that allow storms to undergo RI should aid in improving short-term intensity forecasts in the future.

Acknowledgments. This research was financially supported by National Science Foundation Award OAC-1661663 and Office of Naval Research Awards N000141613033 and N000142012069. We thank the NOAA/Aircraft Operations Center and the Hurricane Research Division of the Atlantic Oceanographic and Meteorological Laboratory for collecting the airborne tail Doppler radar data used for this study. We also thank Rachel Mauk and two anonymous reviewers for comments and suggestions that improved the paper.

Data availability statement. The dataset analyzed from Hurricane Michael in this study can be found online (https://doi.org/ 10.5281/zenodo.5146325).

\section{REFERENCES}

Aircraft Operations Center, 2016: Tropical cyclone operations: Challenges in 2015: New products and services planned for 2016 and 2017. NOAA Office of Marine and Aviation Operations, accessed 29 November 2020, https://www.ofcm.gov/ meetings/TCORF/ihc16/2016presentations.html.

Bell, M. M., 2019: nsf-lrose/lrose-blaze: lrose-blaze-20190105. Accessed 6 January 2019, https://doi.org/10.5281/ZENODO. 2532758.

—, M. T. Montgomery, and K. A. Emanuel, 2012: Air-sea enthalpy and momentum exchange at major hurricane wind speeds observed during CBLAST. J. Atmos. Sci., 69, 31973222, https://doi.org/10.1175/JAS-D-11-0276.1.

— W.-C. Lee, C. A. Wolff, and H. Cai, 2013: A Solo-based automated quality control algorithm for airborne tail Doppler radar data. J. Appl. Meteor. Climatol., 52, 2509-2528, https:// doi.org/10.1175/JAMC-D-12-0283.1.

—_, Beven, J. L., R. Berg, and A. Hagen, 2019: National Hurricane Center tropical cyclone report: Hurricane Michael (7-11 October 2018). NHC Tech. Rep AL142018, 86 pp., https:// www.nhc.noaa.gov/data/tcr/AL142018_Michael.pdf.

Boehm, A. M., and M. M. Bell, 2021: Retrieved thermodynamic structure of Hurricane Rita (2005) from airborne multiDoppler radar data. J. Atmos. Sci., 78, 1583-1605, https://doi. org/10.1175/JAS-D-20-0195.1.

Cai, H., W.-C. Lee, M. M. Bell, C. A. Wolff, X. Tang, and F. Roux, 2018: A generalized navigation correction method for airborne Doppler radar data. J. Atmos. Oceanic Technol., 35, 1999-2017, https://doi.org/10.1175/JTECH-D-18-0028.1.

Cangialosi, J. P., E. Blake, M. DeMaria, A. Penny, A. Latto, E. Rappaport, and V. Tallapragada, 2020: Recent progress in tropical cyclone intensity forecasting at the National Hurricane Center. Wea. Forecasting, 35, 1913-1922, https://doi.org/10.1175/ WAF-D-20-0059.1.
- Cha, T.-Y., M. M. Bell, W.-C. Lee, and A. J. DesRosiers, 2020: Polygonal eyewall asymmetries during the rapid intensification of Hurricane Michael (2018). Geophys. Res. Lett., 47, e2020GL087919, https://doi.org/10.1029/2020GL087919.

Chen, H., and D.-L. Zhang, 2013: On the rapid intensification of Hurricane Wilma (2005). Part II: Convective bursts and the upper-level warm core. J. Atmos. Sci., 70, 146-162, https://doi. org/10.1175/JAS-D-12-062.1.

DeMaria, M., C. R. Sampson, J. A. Knaff, and K. D. Musgrave, 2014: Is tropical cyclone intensity guidance improving? Bull. Amer. Meteor. Soc., 95, 387-398, https://doi.org/10.1175/ BAMS-D-12-00240.1.

DesRosiers, A. J., 2020: Airborne radar quality control and analysis of the rapid intensification of Hurricane Michael (2018). M.S. thesis, Department of Atmospheric Science, Colorado State University, $50 \mathrm{pp}$.

Didlake, A. C., and R. A. Houze Jr., 2011: Kinematics of the secondary eyewall observed in Hurricane Rita (2005). J. Atmos. Sci., 68, 1620-1636, https://doi.org/10.1175/2011JAS3715.1.

Dunion, J. P., 2011: Rewriting the climatology of the tropical North Atlantic and Caribbean Sea atmosphere. J. Climate, 24, 893-908, https://doi.org/10.1175/2010JCLI3496.1.

Eliassen, A., 1951: Slow thermally or frictionally controlled meridional circulation in a circular vortex. Astrophys. Nor., 5, 19-60.

Fischer, M. S., B. H. Tang, K. L. Corbosiero, and C. M. Rozoff, 2018: Normalized convective characteristics of tropical cyclone rapid intensification events in the North Atlantic and eastern North Pacific. Mon. Wea. Rev., 146, 1133-1155, https://doi.org/10.1175/MWR-D-17-0239.1.

Foerster, A. M., and M. M. Bell, 2017: Thermodynamic retrieval in rapidly rotating vortices from multiple-Doppler radar data. J. Atmos. Oceanic Technol., 34, 2353-2374, https://doi.org/10. 1175/JTECH-D-17-0073.1.

Gamache, J. F., J. S. Griffin, P. P. Dodge, and N. F. Griffin, 1997: Evaluation of a fully three-dimensional variational Doppler analysis technique. Preprints, 28th Conf. on Radar Meteorology, Austin, TX, Amer. Meter. Soc., 422-423.

Green, A., S. G. Gopalakrishnan, G. J. Alaka Jr., and S. Chia, 2021: Understanding the role of mean and eddy momentum transport in the rapid intensification of Hurricane Irma (2017) and Hurricane Michael (2018). Atmosphere, 12, 492, https://doi.org/10.3390/atmos12040492.

Hazelton, A. T., and R. E. Hart, 2013: Hurricane eyewall slope as determined from airborne radar reflectivity data: Composites and case studies. Wea. Forecasting, 28, 368-386, https://doi. org/10.1175/WAF-D-12-00037.1.

- X. X. Zhang, S. Gopalakrishnan, W. Ramstrom, F. Marks, and J. A. Zhang, 2020: High-resolution ensemble HFV3 forecasts of Hurricane Michael (2018): Rapid intensification in shear. Mon. Wea. Rev., 148, 2009-2032, https://doi.org/10.1175/ MWR-D-19-0275.1.

Hendricks, E. A., M. T. Montgomery, and C. A. Davis, 2004: The role of "vortical" hot towers in the formation of Tropical Cyclone Diana (1984). J. Atmos. Sci., 61, 1209-1232, https://doi. org/10.1175/1520-0469(2004)061<1209:TROVHT>2.0.CO;2.

— M. S. Peng, B. Fu, and T. Li, 2010: Quantifying environmental control on tropical cyclone intensity change. Mon. Wea. Rev., 138, 3243-3271, https://doi.org/10.1175/2010MWR3185.1.

Hirschberg, P. A., and J. M. Fritsch, 1993: On understanding height tendency. Mon. Wea. Rev., 121, 2646-2661, https://doi. org/10.1175/1520-0493(1993)121<2646:OUHT>2.0.CO;2. 
Hoskins, B. J., and F. P. Bretherton, 1972: Atmospheric frontogenesis models: Mathematical formulation and solution. $J$. Atmos. Sci., 29, 11-37, https://doi.org/10.1175/15200469(1972)029<0011:AFMMFA > 2.0.CO;2.

Houze, R. A., Jr., 2010: Clouds in tropical cyclones. Mon. Wea. Rev., 138, 293-344, https://doi.org/10.1175/2009MWR2989.1.

Kaplan, J., and M. DeMaria, 2003: Large-scale characteristics of rapidly intensifying tropical cyclones in the North Atlantic basin. Wea. Forecasting, 18, 1093-1108, https://doi.org/10. 1175/1520-0434(2003)018<1093:LCORIT>2.0.CO;2.

,$--\longrightarrow$, and J. A. Knaff, 2010: A revised tropical cyclone rapid intensification index for the Atlantic and eastern North Pacific basins. Wea. Forecasting, 25, 220-241, https://doi.org/ 10.1175/2009WAF2222280.1.

Kossin, J. P., and M. D. Eastin, 2001: Two distinct regimes in the kinematic and thermodynamic structure of the hurricane eye and eyewall. J. Atmos. Sci., 58, 1079-1090, https://doi.org/10. 1175/1520-0469(2001)058<1079:TDRITK>2.0.CO;2.

Lee, J.-D., and C.-C. Wu, 2018: The role of polygonal eyewalls in rapid intensification of Typhoon Megi. J. Atmos. Sci., 75, 4175-4199, https://doi.org/10.1175/JAS-D-18-0100.1.

Lorsolo, S., and A. Aksoy, 2012: Wavenumber analysis of azimuthally distributed data: Assessing maximum allowable gap size. Mon. Wea. Rev., 140, 1945-1956, https://doi.org/10.1175/ MWR-D-11-00219.1.

Martinez, J., M. M. Bell, R. Rogers, and J. D. Doyle, 2019: Axisymmetric potential vorticity evolution of Hurricane Patricia (2015). J. Atmos. Sci., 76, 2043-2063, https://doi.org/10.1175/ JAS-D-18-0373.1.

Montgomery, M. T., 2016: Introduction to hurricane dynamics: Tropical cyclone intensification. Advanced Numerical Modeling and Data Assimilation Techniques for Tropical Cyclone Prediction, U. C. Mohanty and S. G. Gopalakrishnan, Eds., Springer, 537-559.

— M. E. Nicholls, T. A. Cram, and A. B. Saunders, 2006: A vortical hot tower route to tropical cyclogenesis. J. Atmos. Sci., 63, 355-386, https://doi.org/10.1175/JAS3604.1.

National Weather Service, 2019: Hurricane Michael hits Georgia. NOAA, accessed 20 June 2020, https://www.weather.gov/ffc/ 2018_hurricane_michael.

Nolan, D. S., Y. Moon, and D. P. Stern, 2007: Tropical cyclone intensification from asymmetric convection: Energetics and efficiency. J. Atmos. Sci., 64, 3377-3405, https://doi.org/10. 1175/JAS3988.1.

Ooyama, K. V., 1969: Numerical simulation of the life cycle of tropical cyclones. J. Atmos. Sci., 26, 3-40, https://doi.org/10. 1175/1520-0469(1969)026<0003:NSOTLC $>2.0 . C O ; 2$.

- 1982: Conceptual evolution of the theory and modeling of the tropical cyclone. J. Meteor. Soc. Japan, 60, 369-380, https://doi.org/10.2151/jmsj1965.60.1_369.

Oye, R., C. Mueller, and S. Smith, 1995: Software for radar translation, visualization, editing, and interpolation. Preprints, 27th Conf. on Radar Meteorology, Vail, CO, Amer. Meteor. Soc., 359-361.

Peng, K., and J. Fang, 2021: Effect of the initial vortex vertical structure on early development of an axisymmetric tropical cyclone. J. Geophys. Res. Atmos., 126, e2020JD033697, https://doi.org/10.1029/2020JD033697.

Reasor, P. D., M. D. Eastin, and J. F. Gamache, 2009: Rapidly intensifying Hurricane Guillermo (1997). Part I: Low- wavenumber structure and evolution. Mon. Wea. Rev., 137, 603-631, https://doi.org/10.1175/2008MWR2487.1.

Rogers, R., 2010: Convective-scale structure and evolution during a high-resolution simulation of tropical cyclone rapid intensification. J. Atmos. Sci., 67, 44-70, https://doi.org/10.1175/ 2009JAS3122.1.

—, P. Reasor, and S. Lorsolo, 2013: Airborne Doppler observations of the inner-core structural differences between intensifying and steady-state tropical cyclones. Mon. Wea. Rev., 141, 2970-2991, https://doi.org/10.1175/MWR-D-12-00357.1.

— , and Coauthors, 2017: Rewriting the tropical record books: The extraordinary intensification of Hurricane Patricia (2015). Bull. Amer. Meteor. Soc., 98, 2091-2112, https://doi. org/10.1175/BAMS-D-16-0039.1.

Schubert, W. H., and J. J. Hack, 1982: Inertial stability and tropical cyclone development. J. Atmos. Sci., 39, 1687-1697, https://doi.org/10.1175/1520-0469(1982)039<1687:

ISATCD $>2.0 . \mathrm{CO} ; 2$.

— , and — 1983: Transformed Eliassen balanced vortex model. J. Atmos. Sci., 40, 1571-1583, https://doi.org/10.1175/ 1520-0469(1983)040<1571:TEBVM > 2.0.CO;2.

—, M. T. Montgomery, R. K. Taft, T. A. Guinn, S. R. Fulton, J. P. Kossin, and J. P. Edwards, 1999: Polygonal eyewalls, asymmetric eye contraction, and potential vorticity mixing in hurricanes. J. Atmos. Sci., 56, 1197-1223, https://doi.org/10. 1175/1520-0469(1999)056<1197:PEAECA > 2.0.CO;2.

- S. R. Fulton, and P. E. Ciesielski, 2017: Elliptic transverse circulation equations for balanced models in a generalized vertical coordinate. 11 pp., https://arxiv.org/abs/1705.05460.

Stern, D. P., and D. S. Nolan, 2009: Reexamining the vertical structure of tangential winds in tropical cyclones: Observations and theory. J. Atmos. Sci., 66, 3579-3600, https://doi.org/ 10.1175/2009JAS2916.1.

$\longrightarrow$, and —, 2012: On the height of the warm core in tropical cyclones. J. Atmos. Sci., 69, 1657-1680, https://doi.org/10.1175/ JAS-D-11-010.1.

- , and F. Zhang, 2016: The warm-core structure of Hurricane Earl (2010). J. Atmos. Sci., 73, 3305-3328, https://doi.org/10. 1175/JAS-D-15-0328.1.

Trabing, B. C., and M. M. Bell, 2020: Understanding error distributions of hurricane intensity forecasts during rapid intensity changes. Wea. Forecasting, 35, 2219-2234, https://doi.org/10. 1175/WAF-D-19-0253.1.

Van Sang, N., R. K. Smith, and M. T. Montgomery, 2008: Tropical-cyclone intensification and predictability in three dimensions. Quart. J. Roy. Meteor. Soc., 137, 563-582, https://doi. org/10.1002/qj.235.

Vigh, J. A., and W. H. Schubert, 2009: Rapid development of the tropical cyclone warm core. J. Atmos. Sci., 66, 3335-3350, https://doi.org/10.1175/2009JAS3092.1.

Wadler, J. B., J. A. Zhang, R. F. Rogers, B. Jaimes, and L. K. Shay, 2020: The rapid intensification of Hurricane Michael (2018): Storm structure and the relationship to environmental and air-sea interactions. Mon. Wea. Rev., 149, 245-267, https://doi.org/10.1175/MWR-D-20-0145.1.

Willoughby, H. E., 1998: Tropical cyclone eye thermodynamics. Mon. Wea. Rev., 126, 3053-3067, https://doi.org/10.1175/15200493(1998)126<3053:TCET>2.0.CO;2. 